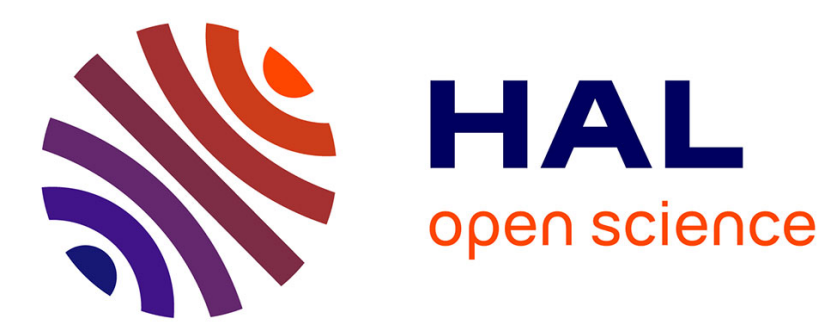

\title{
Hamilton-Jacobi-Bellman Equations with Time-Measurable Data and Infinite Horizon
}

V. Basco, Hélène Frankowska

\section{To cite this version:}

V. Basco, Hélène Frankowska. Hamilton-Jacobi-Bellman Equations with Time-Measurable Data and Infinite Horizon. Nonlinear Differential Equations and Applications, 2019, 26 (7), 10.1007/s00030019-0553-y · hal-02126128

\section{HAL Id: hal-02126128 \\ https://hal.science/hal-02126128}

Submitted on 10 May 2019

HAL is a multi-disciplinary open access archive for the deposit and dissemination of scientific research documents, whether they are published or not. The documents may come from teaching and research institutions in France or abroad, or from public or private research centers.
L'archive ouverte pluridisciplinaire HAL, est destinée au dépôt et à la diffusion de documents scientifiques de niveau recherche, publiés ou non, émanant des établissements d'enseignement et de recherche français ou étrangers, des laboratoires publics ou privés. 


\title{
Hamilton-Jacobi-Bellman Equations with Time-Measurable Data and Infinite Horizon
}

\author{
V. Basco \\ Dipartimento di Matematica, Università di Roma "Tor Vergata", \\ Via della Ricerca Scientifica 1, 00133 Roma, Italy \\ and \\ H. Frankowska, Corresponding author, \\ CNRS, Institut de Mathématiques de Jussieu - Paris Rive Gauche, \\ Sorbonne Université, Case 247, 4 Place Jussieu, 75252 Paris, France
}

July 28,2018

\begin{abstract}
In this paper we investigate the existence and uniqueness of weak solutions of the nonautonomous Hamilton-Jacobi-Bellman equation on the domain $(0, \infty) \times \Omega$. The Hamiltonian is assumed to be merely measurable in time variable and the open set $\Omega$ may be unbounded with nonsmooth boundary. The set $\bar{\Omega}$ is called here a state constraint. When state constraints arise, then classical analysis of Hamilton-JacobiBellman equation lacks appropriate notion of solution because continuous solutions could not exist. In this work we propose a notion of weak solution for which, under a suitable controllability assumption, existence and uniqueness theorems are valid in the class of lower semicontinuous functions vanishing at infinity.
\end{abstract}

\section{Introduction}

The notion of weak (or viscosity) solution to a first-order partial differential equation was introduced in the pioneering works $[8,9,20]$ by Crandall, Evans, and Lions to investigate stationary and evolutionary Hamilton-Jacobi-Bellman (H-J-B) equations, using sub/super solutions involving superdifferentials and subdifferentials of continuous function associated to $C^{1}$ test functions. In particular, they obtained existence and uniqueness results in the class of continuous functions for the Cauchy problem associated to the following H-J-B equation

$$
-\partial_{t} V+\mathscr{H}\left(t, x,-\nabla_{x} V\right)=0 \quad \text { on }(0, T) \times \mathbb{R}^{n},
$$

when the Hamiltonian $\mathscr{H}$ is continuous, while in $[3,26]$ the authors extended the existence results to a large class of continuous Hamiltonians. When the solution is differentiable, then it solves the H-J-B equation also in the classical sense. However, it is well known that such a kind of notion turns out to be quite unsatisfactory for H-J-B equations arising in control theory and the calculus of variations (we refer to $[2,20]$ for further discussions). Indeed, the value function, that is a weak solution of $\mathrm{H}-\mathrm{J}-\mathrm{B}$ equation, loses the differentiability property 
(even in the absence of state constraints) whenever there are multiple optimal solutions at the same initial condition. When additional state constraints are present it also loses its continuity. At most we expect lower semicontinuity of the value function. So, subsequently, the definition of solution was extended to lower semicontinuous functions.

For the Mayer problem (of optimal control theory) free of state constraints involving a continuous cost function and Lipschitz continuous dynamics, the uniqueness of continuous solutions of the associated H-J-B equation can be addressed using the notion of viscosity solution. Further, the definition of solution can be stated equivalently in terms of "normals" to the epigraph and the hypograph of the solution. But, when the dynamics is only measurable in time such equivalence may fail to be true. Nevertheless, the study of uniqueness of weak solutions can be carried out by using the solutions concept from [15], see also Sections 3 and 4 below, based on "normals" to the epigraph. Previously, to deal with Hamiltonian measurable in time, in [17] the author proposed a new notion of weak solution (cfr. [21] for equivalent formulations of such a kind of solutions) in the class of continuous functions, proving, by a blow-up method, the uniqueness and existence in the stationary case on a general open subset of $\mathbb{R}^{n}$ and for the evolutionary case on $(0, \infty) \times \mathbb{R}^{n}$. The $C^{1}$ test functions needed to define such solutions are more complex, involving in addition some integrable mappings. We point out that, under the assumptions that $\mathscr{H}$ is measurable in time, Lipschitz continuous in the space variable, and convex in the last variable, the so called representation theorems (cfr. [16, 22] and the reference therein) associate to the $\mathrm{H}-\mathrm{J}-\mathrm{B}$ equation a control problem in such a way that the value function is a weak solution. This yields an existence result for weak solutions.

To deal with discontinuous solutions, in [18], Ishii introduced the concept of lower and upper semicontinuous envelopes of a function, proving that the upper semicontinuous envelope of the value function of an optimal control problem is the largest upper semicontinuous subsolution and its lower semicontinuous envelope is the smallest lower semicontinuous supersolution. This approach, however, does not ensure the uniqueness of (weak) solutions of the H-J-B equation. On the other hand the upper semicontinuous envelope does not have any meaning in optimal control theory while dealing with minimization problems (the lower semicontinuous envelope determines the value function of the relaxed problem). In $[4,5,10]$ a different concept of solutions was developed for the H-J-B equation associated to the Mayer optimal control problem not involving state constraints, but having a discontinuous cost. In this approach only subdifferentials are involved. In particular, in [10], results are expressed using the Fréchet subdifferentials instead of $C^{1}$ test functions. By [8, Proposition 1.1], Fréchet subdifferentials of continuous functions coincide with those defined in [9] via $C^{1}$ test functions. While investigating in [15] the merely measurable case, it became clear that in order to get uniqueness, it is convenient to replace subdifferentials by normals to the epigraph of solutions. Such "geometric" definition of solution avoids using test functions and allows to have a unified approach to both the continuous and the measurable case.

To deal with state constrained problems, the usual assumptions on data may be not sufficient to derive existence and uniqueness results for the H-J-B equations. In [25] Soner proposed a controllability assumption (the Slatter like assumption) to investigate an autonomous control problem, recovering the continuity of the value function through an inward pointing condition (under the assumption that the set $\Omega$ is bounded with $\partial \Omega \in C^{2}$ ): that is, he assumed that for any $x \in \partial \Omega$ we can find a control $u$ satisfying $\left\langle f(x, u), \nu_{x}\right\rangle<0$, where 
$\nu_{x}$ is the outward unit normal to $\Omega$ at $x$ and $f$ is the dynamics of control system. Such condition implies uniqueness of viscosity solutions. However, it cannot be used for sets with nonsmooth boundary and the boundedness assumption on $\Omega$ may be quite restrictive for many applied models: for instance, macroeconomics models often consider cones as state constraints. To allow nonsmooth boundaries, Ishii and Koike generalized the concept of Soner's condition in the framework of infinite horizon problems and continuous solutions (cfr. [19] and the references therein). More generally, various versions of inward pointing condition are useful to get continuity or Lipschitz continuity of the value function, see for instance [6]. Furthermore, in $[13,14]$ the authors, dealing with paratingent cones and closed set of constraints with possibly empty interior, carry out the analysis under another controllability requirement named outward pointing condition. Such condition ensures, roughly speaking, that any boundary point of $\Omega$ can be reached by trajectories laying in the relative interior of $\Omega$. The outward pointing conditions allow furthermore to use the so called backward neighboring feasible trajectory theorems, fundamental to address the control systems under state constraints. It was used, in particular, in [11], to study an H-J-B equation on finite time interval, when the Hamiltonian is convex and positively homogeneous in the third variable.

We would like to underline here that, in contrast, the inward pointing condition is neither needed, nor well adapted in the context of lower semicontinuous functions because it does not imply uniqueness of solutions to the H-J-B equation unless further regularity assumptions are imposed on the solutions.

The novelty of our work consists in examining the weak solutions (in the sense of Definition 3.2 below) of the H-J-B equation on $(0, \infty) \times \Omega$ (where $\Omega$ is an open subset of $\mathbb{R}^{n}$ with possibly nonsmooth boundary) and with time-measurable Hamiltonian (associated with an infinite horizon optimal control problem). Proofs of uniqueness make use of the geometric properties of epigraphs of such solutions. We recover the uniqueness, from a neighboring feasible trajectory theorem (cfr. [6]) under a backward controllability assumption, in a class of lower semicontinuous functions vanishing at infinity. More precisely, we prove the existence and uniqueness of weak solutions of the following problem

$$
\left\{\begin{array}{l}
-\partial_{t} W+\mathscr{H}\left(t, x,-\nabla_{x} W\right)=0 \quad \text { on }(0, \infty) \times \Omega \\
\lim _{t \rightarrow \infty} \sup _{y \in \operatorname{dom} W(t, \cdot)}|W(t, y)|=0 .
\end{array}\right.
$$

The outline of this paper is as follows. In Section 2 we introduce notations and recall some results from nonsmooth analysis. The main result is stated in Section 3 whose proof is left to Section 4. In the last section we discuss the particular case of the Lipschitz continuous solutions.

\section{Preliminaries}

We denote by $|\cdot|$ and $\langle\cdot, \cdot\rangle$ the Euclidean norm and scalar product in $\mathbb{R}^{k}$, respectively, and by $\mu$ the Lebesgue measure. Let $\left(X,|\cdot|_{X}\right)$ be a normed space, $B(x, \delta)$ stand for the closed ball in $X$ with radius $\delta>0$ centered at $x \in X$ and $\mathbb{B}=B(0,1)$. For a nonempty subset $C \subset X$ we denote the interior of $C$ by int $C$, the boundary of $C$ by $\partial C$, the convex hull of $C$ by co $C$, its closure by $\overline{c o} C$, and the distance from $x \in X$ to $C$ by $d_{C}(x):=\inf \left\{|x-y|_{X}: y \in C\right\}$. If $X=\mathbb{R}^{k}$, in what follows "-" stands for the negative 
polar cone of a set, i.e., $C^{-}=\left\{p \in \mathbb{R}^{k}:\langle p, c\rangle \leqslant 0 \quad \forall c \in C\right\}$. Moreover, we denote the positive polar cone of $C$ by $C^{+}:=-C^{-}$.

Let $I$ and $J$ be two closed intervals in $\mathbb{R}$. We denote by $L^{1}(I ; J)$ the set of all $J$-valued Lebesgue integrable functions on $I$. We say that $f \in L_{\text {loc }}^{1}(I ; J)$ if $f \in L^{1}(K ; J)$ for any compact subset $K \subset I$. We denote by $\mathscr{L}_{\text {loc }}$ the set of all functions $f \in L_{\text {loc }}^{1}\left([0, \infty) ; \mathbb{R}^{+}\right)$ such that $\lim _{\sigma \rightarrow 0} \theta_{f}(\sigma)=0$ where $\theta_{f}(\sigma)=\sup \left\{\int_{J} f(\tau) d \tau: J \subset[0, \infty), \mu(J) \leqslant \sigma\right\}$. We recall that for a function $q \in L_{\mathrm{loc}}^{1}([0, \infty) ; \mathbb{R})$ the integral $\int_{t_{0}}^{\infty} q(s) d s:=\lim _{T \rightarrow \infty} \int_{t_{0}}^{T} q(s) d s$, whenever this limit exists.

Let $D \subset \mathbb{R}^{n}$ be nonempty and $\left\{A_{h}\right\}_{h \in D}$ be a family of nonempty subsets of $\mathbb{R}^{k}$. The upper and lower limits, in the Kuratowski-Painlevé sense, of $A_{h}$ at $h_{0} \in D$ are the closed sets defined respectively by

$\operatorname{Limsup}_{h \rightarrow D_{D} h_{0}} A_{h}=\left\{v \in \mathbb{R}^{k}: \liminf _{h \rightarrow_{D} h_{0}} d_{A_{h}}(v)=0\right\}, \operatorname{Liminf}_{h \rightarrow D} A_{h}=\left\{v \in \mathbb{R}^{k}: \limsup _{h \rightarrow_{D} h_{0}} d_{A_{h}}(v)=0\right\}$.

Consider a nonempty subset $E \subset \mathbb{R}^{k}$ and $x \in \bar{E}$. The contingent cone $T_{E}(x)$ to $E$ at $x$ is defined as the set of all vectors $v \in \mathbb{R}^{k}$ such that $\liminf _{h \rightarrow 0+} \frac{d_{E}(x+h v)}{h}=0$. The limiting normal cone to $E$ at $x$, written $N_{E}(x)$, is defined by $N_{E}(x):=\operatorname{Limsup}_{y \rightarrow E} x T_{E}(y)^{-}$. It is known that $N_{E}(x)^{-} \subset T_{E}(x)$ whenever $E$ is closed. The Clarke tangent cone is defined by $N_{E}(x)^{-}$.

Let $\varphi: \mathbb{R}^{k} \rightarrow \mathbb{R} \cup\{ \pm \infty\}$ be an extended real function. We write $\operatorname{dom} \varphi$ for the domain of $\varphi$, epi $\varphi$ for the epigraph of $\varphi$, and hypo $\varphi$ for the hypograph of $\varphi$. The (Fréchet) subdifferential, respectively the (Fréchet) superdifferential, of $\varphi$ at $x_{0} \in \operatorname{dom} \varphi$ are the possibly empty sets defined by

$$
\partial_{-} \varphi\left(x_{0}\right)=\left\{p \in \mathbb{R}^{k}: \liminf _{x \rightarrow x_{0}} \frac{\varphi(x)-\varphi\left(x_{0}\right)-\left\langle p, x-x_{0}\right\rangle}{\left|x-x_{0}\right|} \geqslant 0\right\}, \partial_{+} \varphi\left(x_{0}\right)=-\partial_{-}(-\varphi)\left(x_{0}\right) .
$$

The contingent epiderivative and the contingent hypoderivative of $\varphi$ at $x_{0} \in \operatorname{dom} \varphi$, in the direction $u \in \mathbb{R}^{k}$, written $D_{\uparrow} \varphi\left(x_{0}\right)(u)$ and $D_{\downarrow} \varphi\left(x_{0}\right)(u)$, respectively, are defined by

$$
D_{\uparrow} \varphi\left(x_{0}\right)(u)=\liminf _{h \rightarrow 0+, u^{\prime} \rightarrow u} \frac{\varphi\left(x_{0}+h u^{\prime}\right)-\varphi\left(x_{0}\right)}{h}, \quad D_{\downarrow} \varphi\left(x_{0}\right)(u)=-D_{\uparrow}(-\varphi)\left(x_{0}\right)(u) .
$$

It is well known that (cfr. [1, Proposition 6.1.4])

$$
\text { epi } D_{\uparrow} \varphi\left(x_{0}\right)=T_{\text {epi } \varphi}\left(x_{0}, \varphi\left(x_{0}\right)\right) \quad \& \quad \text { hypo } D_{\downarrow} \varphi\left(x_{0}\right)=T_{\text {hypo } \varphi}\left(x_{0}, \varphi\left(x_{0}\right)\right) \text {. }
$$

From [7] we know that, for a measurable mapping $\varphi, p \in \partial_{-} \varphi\left(x_{0}\right)$ if and only if there exists a continuous function $\psi: \mathbb{R}^{k} \rightarrow \mathbb{R}$, differentiable at $x_{0}$, such that $\psi(x)<\varphi(x)$ for all $x \neq x_{0}, \varphi\left(x_{0}\right)=\psi\left(x_{0}\right)$, and $\nabla \psi\left(x_{0}\right)=p$. If in addition $\varphi$ is continuous, then $\psi$ can be chosen to be of class $C^{1}$. In this respect for a lower semicontinuous function $\varphi$ the notion of the (Fréchet) subdifferential we consider differs from the one in [9], where only continuous viscosity solutions were investigated and $C^{1}$ support functions were used. Similar remark can be made about superdifferentials.

A set-valued map $F: \mathbb{R}^{k} \rightsquigarrow \mathbb{R}^{n}$ taking nonempty values is said to be upper semicontinuous at $x \in \mathbb{R}^{k}$ if for any $\varepsilon>0$ there exists $\delta>0$ such that $F\left(x^{\prime}\right) \subset F(x)+\varepsilon \mathbb{B}$ for all 
$x^{\prime} \in B(x, \delta)$. If $F$ is upper semicontinuous at every $x$ then it is said to be upper semicontinuous. $F$ is said to be lower semicontinuous at $x \in \mathbb{R}^{k}$ if $\operatorname{Liminf}_{y \rightarrow x} F(y) \subset F(x)$. $F$ is said to be lower semicontinuous if $F$ is lower semicontinuous at every $x \in \mathbb{R}^{k}$. $F$ is called continuous at $x \in \mathbb{R}^{k}$ if it is lower and upper semicontinuous at $x$ and it is continuous if it is continuous at each point $x$.

Definition 2.1. A set-valued map $P: I \rightsquigarrow \mathbb{R}^{k}$ is locally absolutely continuous if it takes nonempty closed images and for any $[S, T] \subset I$, every $\varepsilon>0$, and any compact subset $K \subset \mathbb{R}^{k}$, there exists $\delta>0$ such that for any finite partition $S \leqslant t_{1}<\tau_{1} \leqslant t_{2}<\tau_{2} \leqslant \ldots \leqslant$ $t_{m}<\tau_{m} \leqslant T$ of $[S, T]$

$$
\sum_{i=1}^{m}\left(\tau_{i}-t_{i}\right)<\delta \Longrightarrow \sum_{i=1}^{m} \max \left\{\tilde{d}_{P\left(t_{i}\right)}\left(P\left(\tau_{i}\right) \cap K\right), \tilde{d}_{P\left(\tau_{i}\right)}\left(P\left(t_{i}\right) \cap K\right)\right\}<\varepsilon,
$$

where $\tilde{d}_{E}\left(E^{\prime}\right):=\inf \left\{\beta>0: E^{\prime} \subset E+\beta \mathbb{B}\right\}$ for any $E, E^{\prime} \subset \mathbb{R}^{k}$ (the infimum over an empty set is $+\infty$, by convention).

\section{Main Result}

Consider the infinite horizon optimal control problem

$$
\text { minimize } \int_{t_{0}}^{\infty} L(t, x(t), u(t)) d t
$$

over all the trajectory-control pairs of the state constrained control system on $I=\left[t_{0}, \infty\right)$

$$
\left\{\begin{array}{l}
x^{\prime}(t)=f(t, x(t), u(t)), \quad u(t) \in U(t), \quad \text { for a.e. } t \in I \\
x\left(t_{0}\right)=x_{0}, \quad x(I) \subset A,
\end{array}\right.
$$

where $f:[0, \infty) \times \mathbb{R}^{n} \times \mathbb{R}^{m} \rightarrow \mathbb{R}^{n}$ and $L:[0, \infty) \times \mathbb{R}^{n} \times \mathbb{R}^{m} \rightarrow \mathbb{R}$ are given, $A$ is a nonempty closed subset of $\mathbb{R}^{n}, U:[0, \infty) \rightsquigarrow \mathbb{R}^{m}$ is a Lebesgue measurable set-valued map with closed nonempty images and $\left(t_{0}, x_{0}\right) \in[0, \infty) \times A$ is the initial datum. Every trajectory-control pair $(x(\cdot), u(\cdot))$ that satisfies the state constrained control system $(3)$ on an interval of the form $I=\left[t_{0}, T\right]$ or $I=\left[t_{0}, \infty\right)$ is called feasible on $I$. We refer to such $x(\cdot)$ as a feasible trajectory. The infimum of the cost functional in (2) over all feasible trajectory-control pairs on $I=\left[t_{0}, \infty\right)$, with the initial datum $\left(t_{0}, x_{0}\right)$, is denoted by $V\left(t_{0}, x_{0}\right)$ (if no feasible trajectory-control pair exists at $\left(t_{0}, x_{0}\right)$, or if the integral in $(2)$ is not defined for every feasible pair, we set $\left.V\left(t_{0}, x_{0}\right)=+\infty\right)$. The function $V:[0, \infty) \times A \rightarrow \mathbb{R} \cup\{ \pm \infty\}$ is called the value function of problem (2)-(3). We say that $(\bar{x}(\cdot), \bar{u}(\cdot))$ is an optimal trajectorycontrol pair at $\left(t_{0}, x_{0}\right) \in([0, \infty) \times A) \cap \operatorname{dom} V$ if $V\left(t_{0}, x_{0}\right)=\int_{t_{0}}^{\infty} L(s, \bar{x}(s), \bar{u}(s)) d s$. Finally,

$$
\mathscr{H}(t, x, p):=\sup _{u \in U(t)}(\langle f(t, x, u), p\rangle-L(t, x, u))
$$

is the Hamiltonian function associated to the above problem.

We denote by $(\mathrm{h})$ the following assumptions on $f$ and $L$ :

(h) (i) $\forall x \in \mathbb{R}^{n}$ the mappings $f(\cdot, x, \cdot)$ and $L(\cdot, x, \cdot)$ are Lebesgue-Borel measurable and there exists $\phi \in L^{1}([0, \infty) ; \mathbb{R})$ such that $L(t, x, u) \geqslant \phi(t)$ for a.e. $t \geqslant 0$ and all $(x, u) \in \mathbb{R}^{n} \times \mathbb{R}^{m}$ 
(ii) $\exists c \in L_{\text {loc }}^{1}\left([0, \infty) ; \mathbb{R}^{+}\right)$such that for a.e. $t \geqslant 0$ and for all $x \in \mathbb{R}^{n}, u \in U(t)$

$$
|f(t, x, u)|+|L(t, x, u)| \leqslant c(t)(1+|x|)
$$

(iii) for a.e. $t \geqslant 0$ and all $x \in \mathbb{R}^{n}$, the set-valued map

$$
\mathbb{R}^{n} \ni y \rightsquigarrow\{(f(t, y, u), L(t, y, u)): u \in U(t)\}
$$

is continuous with closed images, and the set

$$
\{(f(t, x, u), L(t, x, u)+r): u \in U(t), r \geqslant 0\}
$$

is convex.

We denote by $(\mathrm{h})^{\prime}$ the assumptions (h) with the further requirement:

(h) $)^{\prime}$ (iv) $\exists k \in L_{\text {loc }}^{1}\left([0, \infty) ; \mathbb{R}^{+}\right)$such that for a.e. $t \geqslant 0$ and for all $x, y \in \mathbb{R}^{n}, u \in U(t)$

$$
|f(t, x, u)-f(t, y, u)|+|L(t, x, u)-L(t, y, u)| \leqslant k(t)|x-y|,
$$

and by $(\mathrm{h})^{\prime \prime}$ the assumptions $(\mathrm{h})^{\prime}$ with the further:

$(\mathrm{h})^{\prime \prime} \quad(\mathrm{v}) \quad k \in \mathscr{L}_{\mathrm{loc}}$ and $\lim \sup _{t \rightarrow \infty} \frac{1}{t} \int_{0}^{t}(c(s)+k(s)) d s<\infty$;

(vi) $\exists q \in \mathscr{L}_{\text {loc }}$ such that for a.e. $t \geqslant 0$

$$
\sup _{u \in U(t)}(|f(t, x, u)|+|L(t, x, u)|) \leqslant q(t), \quad \forall x \in \partial A .
$$

Moreover, we denote by (B) and (OPC) the following assumptions:

(B) $\operatorname{dom} V \neq \emptyset$ and there exist $T>0$ and $\psi \in L^{1}\left([T, \infty) ; \mathbb{R}^{+}\right)$such that for all $\left(t_{0}, x_{0}\right) \in$ $\operatorname{dom} V \cap\left([T, \infty) \times \mathbb{R}^{n}\right)$ and any feasible trajectory-control pair $(x(\cdot), u(\cdot))$ on $I=$ $\left[t_{0}, \infty\right)$, with $x\left(t_{0}\right)=x_{0}$,

$$
|L(t, x(t), u(t))| \leqslant \psi(t) \quad \text { for a.e. } t \geqslant t_{0}
$$

(OPC) there exist $\eta>0, r>0, M \geqslant 0$ such that for a.e. $t>0$ and any $y \in \partial A+\eta \mathbb{B}$, and any $v \in f(t, y, U(t))$, with $\inf _{n \in N_{y, \eta}^{1}}\langle n, v\rangle \leqslant 0$, we can find $w \in f(t, y, U(t)) \cap B(v, M)$ satisfying

$$
\inf _{n \in N_{y, \eta}^{1}}\{\langle n, w\rangle,\langle n, w-v\rangle\} \geqslant r
$$

where $N_{y, \eta}^{1}:=\left\{n \in \partial \mathbb{B}: n \in \overline{\mathrm{co}} N_{A}(x), x \in \partial A \cap B(y, \eta)\right\}$.

We denote by (IPC) the conditions (OPC) in which $f(t, y, U(t))$ is replaced by $-f(t, y, U(t))$.

\section{Remarks 3.1.}

(i) If $L(t, x, u)=e^{-\lambda t} l(t, x, u)$, with $l$ bounded and $\lambda>0$, then (B) is satisfied. 
(ii) If $f(t, \cdot, u)$ and $L(t, \cdot, u)$ are continuous, uniformly in $u \in U(t)$, then the set-valued map in (4) is continuous for a.e. $t \geqslant 0$.

Define the augmented Hamiltonian $H:[0, \infty) \times \mathbb{R}^{n} \times \mathbb{R}^{n} \times \mathbb{R} \rightarrow \mathbb{R}$ by

$$
H(t, x, p, q)=\sup _{u \in U(t)}(\langle f(t, x, u), p\rangle-q L(t, x, u)) .
$$

Definition 3.2. A function $W:[0, \infty) \times A \rightarrow \mathbb{R} \cup\{+\infty\}$ is called a weak (or viscosity) solution of H-J-B equation on $(0, \infty) \times A$ if there exists a set $C^{\prime} \subset(0, \infty)$, with $\mu\left(C^{\prime}\right)=0$, such that for all $(t, x) \in \operatorname{dom} W \cap\left(\left((0, \infty) \backslash C^{\prime}\right) \times \partial A\right)$

$$
-p_{t}+H\left(t, x,-p_{x},-q\right) \geqslant 0 \quad \forall\left(p_{t}, p_{x}, q\right) \in T_{\text {epi } W}(t, x, W(t, x))^{-},
$$

and for all $(t, x) \in \operatorname{dom} W \cap\left(\left((0, \infty) \backslash C^{\prime}\right) \times \operatorname{int} A\right)$

$$
-p_{t}+H\left(t, x,-p_{x},-q\right)=0 \quad \forall\left(p_{t}, p_{x}, q\right) \in T_{\text {epi } W}(t, x, W(t, x))^{-} .
$$

The next theorem ensures the existence and uniqueness of (weak) solutions of the Hamilton-Jacobi-Bellman equation in the class of the lower semicontinuous functions vanishing at infinity.

Theorem 3.3. Assume (h) $)^{\prime \prime}$ and (OPC). Let $W:[0, \infty) \times A \rightarrow \mathbb{R} \cup\{+\infty\}$ be a lower semicontinuous function such that $\operatorname{dom} V(t, \cdot) \subset \operatorname{dom} W(t, \cdot) \neq \emptyset$ for all large $t>0$ and

$$
\lim _{t \rightarrow \infty} \sup _{y \in \operatorname{dom} W(t, \cdot)}|W(t, y)|=0 .
$$

Then the following statements are equivalent:

(i) $W=V$;

(ii) $W$ is a weak solution of $H-J-B$ equation on $(0, \infty) \times A$ and $t \rightsquigarrow$ epi $W(t, \cdot)$ is locally absolutely continuous.

Moreover, if in addition (B) holds true, then $V$ is the unique weak solution satisfying (8) with locally absolutely continuous $t \rightsquigarrow$ epi $V(t, \cdot)$.

\section{Remarks 3.4.}

(i) The proof of Theorem 3.3 given below implies that instead of lower semicontinuity of $W$ we can assume that

$$
\liminf _{s \rightarrow 0+, y \rightarrow{ }_{A} x} W(s, y)=W(0, x) \quad \forall x \in A,
$$

to get the same conclusion as in Theorem 3.3.

(ii) Proposition 4.4- $(v)$ and Remark 4.1- $(i)$ below imply that under the assumptions (h) and (OPC), if $\operatorname{dom}(V) \neq \emptyset$, then the set-valued map $t \rightsquigarrow$ epi $V(t, \cdot)$ is locally absolutely continuous even though $V$ may be discontinuous. 
(iii) From the proof of implication $(i i) \Longrightarrow(i)$ of Theorem 3.3 given in Section 4, it follows that Theorem 3.3 holds true again if the condition (8) is replaced by the weaker requirement

$$
\liminf _{t \rightarrow \infty} \sup _{y \in \operatorname{dom} W(t, \cdot)}|W(t, y)|=0,
$$

and assuming further regularity:

$$
\exists \tau>0: \liminf _{s \rightarrow t-, y \rightarrow \operatorname{int} A} W(s, y)=W(t, x) \quad \forall(t, x) \in(\tau, \infty) \times A .
$$

By Proposition 4.4-(iii) given below and [6, Theorem 2], the value function $V$ satisfies (9) whenever (h) holds true.

(iv) Under the assumption (OPC), if for all large $t \geqslant 0$ and all $x \in A$

$$
\left\{D_{\uparrow} W(t, x)(-1,-v): v \in F(t, x) \cap \operatorname{int}\left(N_{A}(x)^{-}\right)\right\} \cap \mathbb{R} \neq \emptyset,
$$

then condition (9) is satisfied. Indeed, let $\tau>0$ be such that for all $t \in(\tau,+\infty)$ and $x \in A$ there exists $\bar{v} \in F(t, x) \cap \operatorname{int}\left(N_{A}(x)^{-}\right)$with finite $D_{\uparrow} W(t, x)(-1,-\bar{v})$. Then, by [24, Theorem 2], there exists $\eta>0$ such that $x+s w \in A$ for all $w \in B(\bar{v}, \eta)$ and $s \in[0, \eta]$. Now, by the definition of contingent epiderivative there exists $\alpha \in \mathbb{R}$ and $h_{i} \rightarrow 0+, w_{i} \rightarrow \bar{v}$ satisfying $W\left(t-h_{i}, x-h_{i} w_{i}\right)-W(t, x) \leqslant \alpha h_{i}$ for all $i$. Since $x-h_{i} w_{i} \in \operatorname{int} A$ for all large $i$, passing to the lower limit as $i \rightarrow \infty$ and using the lower semicontinuity of $W$, we get (9).

(v) Under the assumptions of Theorem 3.3 and that $f$ and $L$ are continuous, by [23, Theorem 1], the statement $(i)$ of Theorem 3.3 is equivalent to the following: for all $(t, x) \in \operatorname{dom} W \cap((0, \infty) \times \partial A)$

$$
-p_{t}+\mathscr{H}\left(t, x,-p_{x}\right) \geqslant 0 \quad \forall\left(p_{t}, p_{x}\right) \in \partial_{-} W(t, x),
$$

and for all $(t, x) \in \operatorname{dom} W \cap((0, \infty) \times \operatorname{int} A)$

$$
-p_{t}+\mathscr{H}\left(t, x,-p_{x}\right)=0 \quad \forall\left(p_{t}, p_{x}\right) \in \partial_{-} W(t, x) .
$$

\section{Proofs}

We recall first two more definitions. Let $I \subset \mathbb{R}_{+}$be a given interval. Consider a set-valued map $Q: I \rightsquigarrow \mathbb{R}^{k}$ and let $y \in Q(s)$ for some $s \in I, y \in \mathbb{R}^{k}$. The contingent derivative $D Q(s, y)$ of $Q$ at $(s, y)$ is the set-valued map $D Q(s, y): \mathbb{R} \rightsquigarrow \mathbb{R}^{k}$ whose graph is given by graph $D Q(s, y)=T_{\operatorname{graph} Q}(s, y)$. By [1, Proposition 5.1.4],

$$
D Q(s, y)(1)=\left\{v \in \mathbb{R}^{k}: \liminf _{h \rightarrow 0+} \frac{d_{Q(s+h)}(y+h v)}{h}=0\right\} .
$$

For a set-valued map $G: I \times \mathbb{R}^{k} \rightsquigarrow \mathbb{R}^{k}$ taking nonempty values, a locally absolutely continuous function $x: I \rightarrow \mathbb{R}^{k}$ is called a $G$-trajectory if $x^{\prime}(t) \in G(t, x(t))$ for a.e. $t \in I$.

Let us define the set-valued maps $G:[0, \infty) \times \mathbb{R}^{n} \rightsquigarrow \mathbb{R}^{n} \times \mathbb{R}, F:[0, \infty) \times \mathbb{R}^{n} \rightsquigarrow \mathbb{R}^{n}$, and $\tilde{G}:[0, \infty) \times \mathbb{R}^{n} \times \mathbb{R} \rightsquigarrow \mathbb{R}^{n} \times \mathbb{R}$ by

$$
\begin{gathered}
G(t, x):=\{(f(t, x, u),-L(t, x, u)-r): u \in U(t), r \in[0, c(t)(1+|x|)-L(t, x, u)]\}, \\
F(t, x):=f(t, x, U(t)) \quad \& \quad \tilde{G}(t, x, v):=G(t, x) .
\end{gathered}
$$

Remarks below follow directly from the assumptions. 


\section{Remarks 4.1.}

(i) Notice that, if (OPC) holds true, then

$$
-F(t, x) \cap \overline{\operatorname{co}} T_{A}(x) \neq \emptyset \quad \text { for a.e. } t \geqslant 0, \forall x \in A \text {. }
$$

(ii) Let $\left(t_{0}, x_{0}\right) \in[0, \infty) \times \mathbb{R}^{n}$. Then, by Gronwall's lemma and our growth assumptions, any absolutely continuous trajectory $x:\left[t_{0}, \infty\right) \rightarrow \mathbb{R}^{n}$ solving the differential equation in (3) and starting from $x_{0}$ at time $t_{0}$ satisfies $1+|x(t)| \leqslant\left(1+\left|x_{0}\right|\right) e^{\int_{t_{0}}^{t} c(s) d s}$ for all $t \geqslant t_{0}$. In particular, feasible trajectories starting at the same initial condition are uniformly bounded on every finite time interval. Moreover, setting for all $R>0$

$$
\gamma_{R}(t):=(1+R) c(t) e^{\int_{0}^{t} c(s) d s} \quad \forall t \geqslant 0
$$

it follows that $\gamma_{R} \in L_{\text {loc }}^{1}\left([0, \infty) ; \mathbb{R}^{+}\right)$and for any $R>0$, any $\left(t_{0}, x_{0}\right) \in[0, \infty) \times$ $(A \cap B(0, R))$, and any feasible trajectory-control pair $(x(\cdot), u(\cdot))$ on $I=\left[t_{0}, \infty\right)$, with $x\left(t_{0}\right)=x_{0}$, we have

$$
|f(t, x(t), u(t))|+|L(t, x(t), u(t))| \leqslant \gamma_{R}(t) \quad \text { for a.e. } t \geqslant t_{0}
$$

(iii) To apply the results from [15, Sections 2 and 4] we extend them to maps with sublinear growth in the following way: letting $R>0$ and $T>0$, the set-valued map $G_{*}$ : $[0, T] \times \mathbb{R}^{n+1} \rightsquigarrow \mathbb{R}^{n+1}$ defined by $G_{*}(t, X)=\tilde{G}(t, X)$ for any $(t, X) \in[0, T] \times B(0, M)$ and $G_{*}(t, X)=\tilde{G}(t, \pi(X))$ for any $(t, X) \in[0, T] \times\left(\mathbb{R}^{n+1} \backslash B(0, M)\right)$, where $\pi(\cdot)$ stands for the projection operator onto $B(0, M)$ and $M=R+2 \int_{0}^{T} \gamma_{R}(s) d s$, satisfies

$$
\sup _{v \in G_{*}(t, X), X \in \mathbb{R}^{n+1}}|v| \leqslant 2 \gamma_{R}(t) \quad \text { for a.e. } t \in[0, T] .
$$

Thus, $X:\left[t_{0}, T\right] \rightarrow \mathbb{R}^{n+1}$, with $X\left(t_{0}\right) \in B(0, R)$, is a $G_{*}$-trajectory if and only if it is $\tilde{G}$-trajectory on $\left[t_{0}, T\right]$.

(iv) Since we assume that the set-valued map $U(\cdot)$ takes nonempty images, so are $G(\cdot)$ and $F(\cdot)$. Moreover, (OPC) implies that $A$ is the closure of its interior. Similarly, for (IPC).

Proposition 4.2. Under assumption (h), for all $x \in \mathbb{R}^{n}$ the set-valued maps $F(\cdot, x)$ and $G(\cdot, x)$ are Lebesgue measurable. Furthermore, for a.e. $t \geqslant 0$ the set-valued maps $G(t, \cdot)$ and $F(t, \cdot)$ are continuous with closed convex images.

Proof. The first statement follows from assumption (h)-(i). Notice that, by (h)-(iii), for a.e. $\quad t \geqslant 0, F(t, \cdot)$ is continuous and $F(t, x)$ is closed convex, since it is the projection of the closed set in (4) and the convex set in (5). Now, consider $t \geqslant 0$ and $x \in$ $\mathbb{R}^{n}$ such that $\{(f(t, x, u), L(t, x, u)): u \in U(t)\}$ is closed and (h)-(ii) holds true. Let $\left(f\left(t, x, u_{k}\right),-L\left(t, x, u_{k}\right)-r_{k}\right) \rightarrow(a, b) \in \mathbb{R}^{n} \times \mathbb{R}$ with $u_{k} \in U(t)$ and $r_{k} \in[0, c(t)(1+|x|)-$ $\left.L\left(t, x, u_{k}\right)\right]$ for all $k$. Since $\left\{L\left(t, x, u_{k}\right)\right\}_{k}$ is bounded we deduce that $\left\{r_{k}\right\}_{k}$ is bounded. So, we may assume that $r_{k} \rightarrow r \geqslant 0$. Then $\left(f\left(t, x, u_{k}\right), L\left(t, x, u_{k}\right)\right) \rightarrow(a,-b-r)$, and, by closedness, there exists $u \in U(t)$ such that $a=f(t, x, u)$ and $-b-r=L(t, x, u)$. This proves that $G(t, x)$ is closed. 
Now, let $t \in[0, \infty)$ be such that $x \rightsquigarrow\{(f(t, x, u), L(t, x, u)): u \in U(t)\}$ is continuous. Then $x \rightsquigarrow G_{1}(t, x):=\{(f(t, x, u),-L(t, x, u)): u \in U(t)\}$ and $x \rightsquigarrow G_{2}(t, x):=$ $\left\{(f(t, x, u),-c(t)(1+|x|): u \in U(t)\}\right.$ are continuous. Thus $x \rightsquigarrow G_{1}(t, x) \cup G_{2}(t, x)$ is continuous, and it follows that $\Gamma: x \rightsquigarrow \overline{\mathrm{co}}\left(G_{1}(t, x) \cup G_{2}(t, x)\right)$ is continuous too (cfr. [1]). Since $G(t, x)=\Gamma(x)$, we deduce that $G(t, x)$ is convex and $G(t, \cdot)$ is continuous.

In the same way as the proof of continuity of $G(t, \cdot)$ in the above Proposition, we show the next result.

Proposition 4.3. If $(\mathrm{h})^{\prime}$ holds true, then for a.e. $t \geqslant 0$ the set-valued map $G(t, \cdot)$ is Lipschitz continuous with constant $k(t)+c(t)$.

The following Proposition summarizes some properties satisfied by the value function $V$.

Proposition 4.4. Assume (h). Then

(i) $V$ is lower semicontinuous and for any $(t, x) \in \operatorname{dom} V$ there exists an optimal trajectorycontrol pair $(\bar{x}(\cdot), \bar{u}(\cdot))$ at $(t, x)$. Moreover, for any $x \in A$

$$
\liminf _{s \rightarrow 0+, y \rightarrow{ }_{A} x} V(s, y)=V(0, x)
$$

(ii) there exists a set $C \subset[0, \infty)$, with $\mu(C)=0$, such that for any $(t, x) \in \operatorname{dom} V \cap$ $(([0, \infty) \backslash C) \times A)$

$$
\exists \bar{u} \in U(t), \quad D_{\uparrow} V(t, x)(1, f(t, x, \bar{u})) \leqslant-L(t, x, \bar{u}) ;
$$

(iii) there exists a set $C^{\prime} \subset(0, \infty)$, with $\mu\left(C^{\prime}\right)=0$, such that for any $(t, x) \in \operatorname{dom} V \cap$ $\left(\left((0, \infty) \backslash C^{\prime}\right) \times \operatorname{int} A\right)$

$$
\forall u \in U(t), \quad D_{\uparrow} V(t, x)(-1,-f(t, x, u)) \leqslant L(t, x, u) ;
$$

(iv) there exists a set $C^{\prime \prime} \subset(0, \infty)$, with $\mu\left(C^{\prime \prime}\right)=0$, such that for any $(t, x) \in \operatorname{dom} V \cap$ $\left(\left((0, \infty) \backslash C^{\prime \prime}\right) \times \operatorname{int} A\right)$

$$
\forall u \in U(t), \quad-L(t, x, u) \leqslant D_{\downarrow} V(t, x)(1, f(t, x, u)) ;
$$

(v) if (11) holds true and $\operatorname{dom} V \neq \emptyset$ then $t \rightsquigarrow \operatorname{epi} V(t, \cdot)$ is locally absolutely continuous.

Remark 4.5. We would like to underline that the local absolute continuity of $t \rightsquigarrow$ epi $V(t, \cdot)$ does not yield local absolute continuity or even continuity of $V(\cdot, x)$. It implies however that $\liminf _{s \rightarrow t_{0}-, x \rightarrow{ }_{A} x_{0}} V(s, x)=V\left(t_{0}, x_{0}\right)$ for all $\left(t_{0}, x_{0}\right) \in \operatorname{dom} V \cap((0, \infty) \times A)$ and that $\liminf _{s \rightarrow t_{0}+, x \rightarrow{ }_{A} x_{0}} V(s, x)=V\left(t_{0}, x_{0}\right)$ for all $\left(t_{0}, x_{0}\right) \in \operatorname{dom} V \cap([0, \infty) \times A)$.

Proof of Proposition 4.4. The first two statements in $(i)$ are well known. Let $x \in A$. If $V(0, x)=+\infty$ then, since $V$ is lower semicontinuous, (12) holds true. Suppose next that $(0, x) \in \operatorname{dom} V$. Consider an optimal trajectory-control pair $(\bar{x}(\cdot), \bar{u}(\cdot))$ at $(0, x)$. Then, by the dynamic programming principle, for all $s \geqslant 0$

$$
V(s, \bar{x}(s))=V(0, x)-\int_{0}^{s} L(\xi, \bar{x}(\xi), \bar{u}(\xi)) d \xi .
$$


So, $\lim _{s \rightarrow 0+} V(s, \bar{x}(s))=V(0, x)$. The lower semicontinuity of $V$ ends the proof of $(i)$.

To prove $(i i)$, let $j \in \mathbb{N}^{+}$. From [15, Corollary 2.7] applied to the set-valued map $\tilde{G}$, there exists a set $C_{j} \subset[0, j]$, with $\mu\left(C_{j}\right)=0$, such that for any $\left(t_{0}, x_{0}\right) \in\left(\left([0, j] \backslash C_{j}\right) \times A\right) \cap \operatorname{dom} V$ and any optimal trajectory-control pair $(\bar{x}(\cdot), \bar{u}(\cdot))$ at $\left(t_{0}, x_{0}\right)$,

$$
\emptyset \neq \operatorname{Limsup}_{\xi \rightarrow t_{0}+}\left\{\frac{1}{\xi-t_{0}}\left(\bar{x}(\xi)-x_{0},-\int_{t_{0}}^{\xi} L(s, \bar{x}(s), \bar{u}(s)) d s\right)\right\} \subset G\left(t_{0}, x_{0}\right) .
$$

Furthermore, by the dynamic programming principle, for all $t \geqslant t_{0}$

$$
V(t, \bar{x}(t))-V\left(t_{0}, x_{0}\right)=-\int_{t_{0}}^{t} L(s, \bar{x}(s), \bar{u}(s)) d s .
$$

So, dividing by $t-t_{0}$ this equality, passing to the lower limit as $t \rightarrow t_{0}+$, and using (16), we get (13). Then (ii) follows setting $C=\cup_{j \in \mathbb{N}^{+}} C_{j}$.

We prove next (iii). Let $j \in \mathbb{N}^{+}$. From Remark 4.1-(iii), [15, Theorem 2.9] applied to the set-valued map $-\tilde{G}(j-\cdot, \cdot, \cdot)$, and from the measurable selection theorem, we can find a subset $C_{j}^{\prime} \subset[1 / j, j]$, with $\mu\left(C_{j}^{\prime}\right)=0$, such that for any $\left(t_{0}, x_{0}\right) \in\left((1 / j, j] \backslash C_{j}^{\prime}\right) \times \operatorname{int} A$ and any $u_{0} \in U\left(t_{0}\right)$ there exist $t_{1} \in\left[1 / j, t_{0}\right)$ and a trajectory-control pair $((x, v),(u, r))(\cdot)$ satisfying

$$
\begin{cases}\left(x^{\prime}(t), v^{\prime}(t)\right)=(f(t, x(t), u(t)),-L(t, x(t), u(t))-r(t)) & \text { for a.e. } t \in\left[t_{1}, t_{0}\right] \\ \left(x\left(t_{0}\right), v\left(t_{0}\right)\right)=\left(x_{0}, 0\right) & \\ u(t) \in U(t), r(t) \in[0, c(t)(1+|x(t)|)-L(t, x(t), u(t))] & \text { for a.e. } t \in\left[t_{1}, t_{0}\right] \\ \left(x^{\prime}\left(t_{0}\right), v^{\prime}\left(t_{0}\right)\right)=\left(f\left(t_{0}, x_{0}, u_{0}\right),-L\left(t_{0}, x_{0}, u_{0}\right)\right), & \end{cases}
$$

and $x\left(\left[t_{1}, t_{0}\right]\right) \subset A$. Hence, if $\left(t_{0}, x_{0}\right) \in \operatorname{dom} V$, by the dynamic programming principle it follows that for all $s \in\left[t_{1}, t_{0}\right]$

$$
\frac{V(s, x(s))-V\left(t_{0}, x_{0}\right)}{t_{0}-s} \leqslant \frac{1}{t_{0}-s}\left(v(s)-v\left(t_{0}\right)\right) .
$$

Passing to the lower limit when $s \rightarrow t_{0}-$, we have that

$$
D_{\uparrow} V\left(t_{0}, x_{0}\right)\left(-1,-f\left(t_{0}, x_{0}, u_{0}\right)\right) \leqslant L\left(t_{0}, x_{0}, u_{0}\right) .
$$

Since $u_{0} \in U\left(t_{0}\right)$ is arbitrary and setting $C^{\prime}=\cup_{j \in \mathbb{N}^{+}} C_{j}^{\prime}$, we get (iii). Moreover, arguing in a similar way, we deduce that $(i v)$ holds true as well.

Now, assume (11) and that $\operatorname{dom} V \neq \emptyset$. Notice that the value function $V$ is bounded from the below and since it is lower semicontinuous, $t \rightsquigarrow \operatorname{epi} V(t, \cdot)$ takes closed images. Let $(\bar{t}, \bar{x}) \in \operatorname{dom} V$. Then, by the dynamic programming principle, it follows that the set-valued map $t \rightsquigarrow$ epi $V(t, \cdot)$ takes nonempty values on $[\bar{t}, \infty)$. If $\bar{t}>0$, consider $\tau \in[0, \bar{t})$. From (11) and (10), it follows that $-F(t, x) \cap D P(t, x)(1) \neq \emptyset$ for a.e. $t \in(\tau, \bar{t}$ and all $x \in A$, where $P(\cdot) \equiv A$. Hence, Remark 4.1-(iii), the viability theorem [15, Theorem 4.2] applied to the set-valued map $-F(\bar{t}-\cdot, \cdot)$, and the measurable selection theorem, imply that there exists a feasible trajectory-control pair $(\tilde{x}(\cdot), \tilde{u}(\cdot))$ on $I=[\tau, \bar{t}]$ satisfying $\tilde{x}(\bar{t})=\bar{x}$. So, applying again the dynamic programming principle and since $\tau \in[0, \bar{t})$ is arbitrary, it follows that $t \rightsquigarrow \operatorname{epi} V(t, \cdot)$ takes nonempty values on $[0, \bar{t}]$. Now, fix $0 \leqslant t_{1} \leqslant t_{0}$. Let $K \subset \mathbb{R}^{n+1}$ be a 
nonempty compact subset, $\left(x_{1}, v_{1}\right) \in \operatorname{epi} V\left(t_{1}, \cdot\right) \cap K$, and put $R=\max _{y \in K}|y|$. Consider an optimal trajectory-control pair $(\bar{x}(\cdot), \bar{u}(\cdot))$ at $\left(t_{1}, x_{1}\right)$. Then

$$
\begin{aligned}
V\left(t_{1}, x_{1}\right)-\int_{t_{1}}^{t_{0}} \phi(s) d s & =\int_{t_{1}}^{\infty} L(s, \bar{x}(s), \bar{u}(s)) d s-\int_{t_{1}}^{t_{0}} \phi(s) d s \\
& \geqslant \int_{t_{0}}^{\infty} L(s, \bar{x}(s), \bar{u}(s)) d s=V\left(t_{0}, \bar{x}\left(t_{0}\right)\right) .
\end{aligned}
$$

Since $v_{1} \geqslant V\left(t_{1}, x_{1}\right)$ we get $\left(\bar{x}\left(t_{0}\right), v_{1}-\int_{t_{1}}^{t_{0}} \phi(s) d s\right) \in \operatorname{epi} V\left(t_{0}, \cdot\right)$. Hence we deduce that

$$
\left(x_{1}, v_{1}\right) \in \operatorname{epi} V\left(t_{0}, \cdot\right)+\int_{t_{1}}^{t_{0}}\left(\gamma_{R}(s)+|\phi(s)|\right) d s \mathbb{B} .
$$

On the other hand, let $\left(x_{0}, v_{0}\right) \in \operatorname{epi} V\left(t_{0}, \cdot\right) \cap K$. Applying again Remark 4.1-(iii), the viability theorem [15, Theorem 4.2], and the measurable selection theorem, we deduce that there exists a feasible trajectory-control pair $(\tilde{x}(\cdot), \tilde{u}(\cdot))$ on $I=\left[t_{1}, t_{0}\right]$ satisfying $\tilde{x}\left(t_{0}\right)=x_{0}$. So, by the dynamic programming principle, we get $V\left(t_{1}, \tilde{x}\left(t_{1}\right)\right) \leqslant V\left(t_{0}, x_{0}\right)+$ $\int_{t_{1}}^{t_{0}} L(s, \tilde{x}(s), \tilde{u}(s)) d s \leqslant v_{0}+\int_{t_{1}}^{t_{0}} \gamma_{R}(s) d s$, i.e., $\left(\tilde{x}\left(t_{1}\right), v_{0}+\int_{t_{1}}^{t_{0}} \gamma_{R}(s) d s\right) \in \operatorname{epi} V\left(t_{1}, \cdot\right)$. Finally, since $\left(x_{0}, v_{0}\right)=\left(\tilde{x}\left(t_{1}\right), v_{0}+\int_{t_{1}}^{t_{0}} \gamma_{R}(s) d s\right)+\left(x_{0}-\tilde{x}\left(t_{1}\right),-\int_{t_{1}}^{t_{0}} \gamma_{R}(s) d s\right)$, we conclude

$$
\left(x_{0}, v_{0}\right) \in \operatorname{epi} V\left(t_{1}, \cdot\right)+2 \int_{t_{1}}^{t_{0}} \gamma_{R}(s) d s \mathbb{B},
$$

and so $(v)$ follows.

The proof of the following lemma can be found in the Appendix.

Lemma 4.6. Assume $(\mathrm{h})^{\prime}$. Let $W:[0, \infty) \times A \rightarrow \mathbb{R} \cup\{+\infty\}$ be such that $t \rightsquigarrow$ epi $W(t, \cdot)$ is locally absolutely continuous. If there exists a set $C^{\prime} \subset(0, \infty)$, with $\mu\left(C^{\prime}\right)=0$, such that for all $(t, x) \in \operatorname{dom} W \cap\left(\left((0, \infty) \backslash C^{\prime}\right) \times \operatorname{int} A\right)$

$$
-p_{t}+H\left(t, x,-p_{x},-q\right) \leqslant 0 \quad \forall\left(p_{t}, p_{x}, q\right) \in T_{\text {epi } W}(t, x, W(t, x))^{-},
$$

then for all $0<\tau_{0}<\tau_{1}$ and any feasible trajectory-control pair $(x(\cdot), u(\cdot))$ on $I=\left[\tau_{0}, \tau_{1}\right]$, with $x\left(\left[\tau_{0}, \tau_{1}\right]\right) \subset \operatorname{int} A$ and $\left(\tau_{1}, x\left(\tau_{1}\right)\right) \in \operatorname{dom} W$, the solution $w(\cdot)$ of

$$
\left\{\begin{array}{l}
w^{\prime}(t)=-L(t, x(t), u(t)) \quad \text { for a.e. } t \in\left[\tau_{0}, \tau_{1}\right] \\
w\left(\tau_{1}\right)=W\left(\tau_{1}, x\left(\tau_{1}\right)\right)
\end{array}\right.
$$

satisfies

$$
(x(t), w(t)) \in \operatorname{epi} W(t, \cdot) \quad \forall t \in\left[\tau_{0}, \tau_{1}\right] .
$$

Remark 4.7. By the definition of local absolutely continuity, our assumption implies that epi $W(t, \cdot)$ is a nonempty closed set for all $t \geqslant 0$. In particular, dom $W(t, \cdot) \neq \emptyset$ and $W(t, \cdot)$ is lower semicontinuous for all $t \geqslant 0$.

Arguing in analogous way as in the proof of Lemma 4.6, we have the following result involving the hypograph: 
Lemma 4.8. Assume $(\mathrm{h})^{\prime}$. Let $W:[0, \infty) \times A \rightarrow \mathbb{R} \cup\{+\infty\}$ be such that

$$
t \rightsquigarrow\{(x, v): v \leqslant W(t, x) \neq+\infty\}
$$

is locally absolutely continuous. If there exists a set $C^{\prime} \subset(0, \infty)$, with $\mu\left(C^{\prime}\right)=0$, such that for all $(t, x) \in \operatorname{dom} W \cap\left(\left((0, \infty) \backslash C^{\prime}\right) \times \operatorname{int} A\right)$

$$
-p_{t}+H\left(t, x,-p_{x},-q\right) \leqslant 0 \quad \forall\left(p_{t}, p_{x}, q\right) \in T_{\text {hypo } W}(t, x, W(t, x))^{+},
$$

then for all $0<\tau_{0}<\tau_{1}$ and any feasible trajectory-control pair $(x(\cdot), u(\cdot))$ on $I=\left[\tau_{0}, \tau_{1}\right]$, with $x\left(\left[\tau_{0}, \tau_{1}\right]\right) \subset \operatorname{int} A$ and $\left(\tau_{0}, x\left(\tau_{0}\right)\right) \in \operatorname{dom} W$, the solution $w(\cdot)$ of

$$
\left\{\begin{array}{l}
w^{\prime}(t)=-L(t, x(t), u(t)) \quad \text { for a.e. } t \in\left[\tau_{0}, \tau_{1}\right] \\
w\left(\tau_{0}\right)=W\left(\tau_{0}, x\left(\tau_{0}\right)\right)
\end{array}\right.
$$

satisfies

$$
(x(t), w(t)) \in \operatorname{hypo} W(t, \cdot) \quad \forall t \in\left[\tau_{0}, \tau_{1}\right] .
$$

Proposition 4.9. Let $W:[0, \infty) \times A \rightarrow \mathbb{R} \cup\{+\infty\}$ be such that $t \rightsquigarrow$ epi $W(t, \cdot)$ is locally absolutely continuous.

(i) If (h)(i)-(ii) hold true and $G(t, \cdot)$ is upper semicontinuous, with closed convex images, for a.e. $t \geqslant 0$, then the following two statements are equivalent:

(a) there exists a set $C \subset(0, \infty)$, with $\mu(C)=0$, such that for all $(t, x) \in \operatorname{dom} W \cap$ $(((0, \infty) \backslash C) \times A)$

$$
\exists \bar{u} \in U(t), \quad D_{\uparrow} W(t, x)(1, f(t, x, \bar{u})) \leqslant-L(t, x, \bar{u}) ;
$$

(b) there exists a set $C^{\prime} \subset(0, \infty)$, with $\mu\left(C^{\prime}\right)=0$, such that for all $(t, x) \in \operatorname{dom} W \cap$ $\left(\left((0, \infty) \backslash C^{\prime}\right) \times A\right)$

$$
-p_{t}+H\left(t, x,-p_{x},-q\right) \geqslant 0 \quad \forall\left(p_{t}, p_{x}, q\right) \in T_{\text {epi } W}(t, x, W(t, x))^{-} .
$$

(ii) If (h)' holds true, then the following two statements are equivalent:

$(a)^{\prime}$ there exists a set $C \subset(0, \infty)$, with $\mu(C)=0$, such that for all $(t, x) \in \operatorname{dom} W \cap$ $(((0, \infty) \backslash C) \times \operatorname{int} A)$

$$
\forall u \in U(t), \quad D_{\uparrow} W(t, x)(-1,-f(t, x, u)) \leqslant L(t, x, u)
$$

$(b)^{\prime}$ there exists a set $C^{\prime} \subset(0, \infty)$, with $\mu\left(C^{\prime}\right)=0$, such that for all $(t, x) \in \operatorname{dom} W \cap$ $\left(\left((0, \infty) \backslash C^{\prime}\right) \times \operatorname{int} A\right)$

$$
-p_{t}+H\left(t, x,-p_{x},-q\right) \leqslant 0 \quad \forall\left(p_{t}, p_{x}, q\right) \in T_{\mathrm{epi} W}(t, x, W(t, x))^{-} .
$$


Proof. We prove $(i)$. Suppose $(a)$. Fix $(t, x) \in \operatorname{dom} W \cap(((0, \infty) \backslash C) \times A)$ and let $\left(p_{t}, p_{x}, q\right) \in T_{\text {epi } W}(t, x, W(t, x))^{-}$. From (1) and (21), we have $(1, f(t, x, \bar{u}),-L(t, x, \bar{u})) \in$ $T_{\text {epi } W}(t, x, W(t, x))$. Thus $p_{t}+\left\langle p_{x}, f(t, x, \bar{u})\right\rangle-q L(t, x, \bar{u}) \leqslant 0$, and so

$$
-p_{t}+H\left(t, x,-p_{x},-q\right) \geqslant 0
$$

Suppose next that $(b)$ is satisfied and let $j \in \mathbb{N}^{+}$. By the separation theorem, (b) implies that

$$
(\{1\} \times G(t, x)) \cap \overline{\mathrm{co}} T_{\text {epi } W}(t, x, W(t, x)) \neq \emptyset
$$

for all $(t, x) \in \operatorname{dom} W \cap\left(\left((0, j) \backslash C^{\prime}\right) \times A\right)$. By [15, Corollary 2.7] and [12, Corollary 3.2], for a set $C_{j} \subset[0, j]$, with $\mu\left(C_{j}\right)=0$, and for all $t_{0} \in[0, j] \backslash C_{j}$ and all $\left(x_{0}, v_{0}\right) \in P\left(t_{0}\right):=$ epi $W\left(t_{0}, \cdot\right)$ there exists a $\tilde{G}$-trajectory $(x, v)(\cdot)$ on $\left[t_{0}, j\right]$, with $\left(x\left(t_{0}\right), v\left(t_{0}\right)\right)=\left(x_{0}, v_{0}\right)$, satisfying $(x, v)(t) \in P(t)$ for all $t \in\left[t_{0}, j\right]$ and

$$
\emptyset \neq \operatorname{Limsup}_{\xi \rightarrow t_{0}+}\left\{\frac{1}{\xi-t_{0}}\left(x(\xi)-x_{0}, v(\xi)-v\left(t_{0}\right)\right)\right\} \subset G\left(t_{0}, x_{0}\right) .
$$

Taking $v_{0}=W\left(t_{0}, x_{0}\right)$, by the measurable selection theorem we conclude that there exist two measurable functions $u(\cdot)$ and $r(\cdot)$, with $u(t) \in U(t)$ and $r(t) \in[0, c(t)(1+|x(t)|)-$ $L(t, x(t), u(t))]$ for a.e. $t \in\left[t_{0}, j\right]$, such that $v(t)=W\left(t_{0}, x_{0}\right)-\int_{t_{0}}^{t} L(s, x(s), u(s)) d s-$ $\int_{t_{0}}^{t} r(s) d s \geqslant W(t, x(t))$ for any $t \in\left[t_{0}, j\right]$. Then

$$
v(t)-v\left(t_{0}\right) \geqslant W(t, x(t))-W\left(t_{0}, x_{0}\right) \quad \forall t \in\left[t_{0}, j\right] .
$$

So, dividing by $t-t_{0}$ the last inequality and passing to the lower limit as $t \rightarrow t_{0}+,(21)$ follows for $C=\cup_{j \in \mathbb{N}^{+}} C_{j}$.

To prove $(i i)$, suppose that $(\mathrm{h})^{\prime}$ holds true. Assuming $(a)^{\prime}$ and arguing similarly to (i), we can conclude that there exists $C^{\prime} \subset(0, \infty)$, with $\mu\left(C^{\prime}\right)=0$, such that $-p_{t}+$ $H\left(t, x,-p_{x},-q\right) \leqslant 0$ for all $\left(p_{t}, p_{x}, q\right) \in T_{\text {epi } W}(t, x, W(t, x))^{-}$and all $(t, x) \in \operatorname{dom} W \cap$ $\left(\left((0, \infty) \backslash C^{\prime}\right) \times \operatorname{int} A\right)$. Now, assume $(b)^{\prime}$ and let $j \in \mathbb{N}^{+}$. From Remark 4.1-(iii), Proposition 4.2 , and [15, Theorem 2.9] applied to the set-valued map $\tilde{G}(j-\cdot, \cdot)$, and the measurable selection theorem, we can find a subset $C_{j} \subset[1 / j, j]$, with $\mu\left(C_{j}\right)=0$, such that for any $\left(t_{0}, x_{0}\right) \in\left((1 / j, j] \backslash C_{j}\right) \times \operatorname{int} A$ and any $u_{0} \in U\left(t_{0}\right)$ there exist $t_{1} \in\left[1 / j, t_{0}\right)$ and a trajectorycontrol pair $((x, v),(u, r))(\cdot)$ satisfying $(17)$ and $x\left(\left[t_{1}, t_{0}\right]\right) \subset \operatorname{int} A$. From Lemma 4.6 we get

$$
v(s)-v\left(t_{0}\right) \geqslant W(s, x(s))-W\left(t_{0}, x\left(t_{0}\right)\right) \quad \forall s \in\left[t_{1}, t_{0}\right] .
$$

Hence, dividing by $t_{0}-s$, passing to the lower limit as $s \rightarrow t_{0}-$, and since $u_{0} \in U\left(t_{0}\right)$ is arbitrary, we have (22) after taking $C=\cup_{j \in \mathbb{N}^{+}} C_{j}$.

Proof of Theorem 3.3. By Proposition 4.9, (ii) is equivalent to the following:

(iii) there exists a set $C \subset(0, \infty)$, with $\mu(C)=0$, such that for all $(t, x) \in \operatorname{dom} W \cap$ $(((0, \infty) \backslash C) \times A)$

$$
\exists \bar{u} \in U(t), \quad D_{\uparrow} W(t, x)(1, f(t, x, \bar{u})) \leqslant-L(t, x, \bar{u}),
$$


for all $(t, x) \in \operatorname{dom} W \cap(((0, \infty) \backslash C) \times \operatorname{int} A)$

$$
\forall u \in U(t), \quad D_{\uparrow} W(t, x)(-1,-f(t, x, u)) \leqslant L(t, x, u),
$$

and $t \rightsquigarrow$ epi $W(t, \cdot)$ is locally absolutely continuous.

Furthermore, the implication $(i) \Longrightarrow($ iii $)$ follows from Proposition 4.4. We have to prove $(i i) \Longrightarrow(i)$. Fix $\left(t_{0}, x_{0}\right) \in(0, \infty) \times A$.

We first show that $W\left(t_{0}, x_{0}\right) \geqslant V\left(t_{0}, x_{0}\right)$. If $W\left(t_{0}, x_{0}\right)=+\infty$, then $W\left(t_{0}, x_{0}\right) \geqslant$ $V\left(t_{0}, x_{0}\right)$. Suppose next that $\left(t_{0}, x_{0}\right) \in \operatorname{dom} W$. From the separation theorem and (6) we deduce (23) for all $(t, x) \in \operatorname{dom} W \cap\left(\left([0, \infty) \backslash C^{\prime}\right) \times A\right)$. By [12, Corollary 3.2] applied with $P(t)=$ epi $W(t, \cdot)$ there exists an absolutely continuous trajectory $X_{0}(\cdot)=\left(x_{0}(\cdot), v_{0}(\cdot)\right)$ solving

$$
\left\{\begin{array}{l}
X^{\prime}(t) \in \tilde{G}(t, X(t)) \quad \text { for a.e. } t \in\left[t_{0}, t_{0}+1\right], X(t)=(x(t), v(t)) \\
x\left(\left[t_{0}, t_{0}+1\right]\right) \subset A \\
x\left(t_{0}\right)=x_{0}, v\left(t_{0}\right)=W\left(t_{0}, x_{0}\right) \\
v(t) \geqslant W(t, x(t)) \quad \forall t \in\left[t_{0}, t_{0}+1\right] .
\end{array}\right.
$$

We claim that for any $j \in \mathbb{N}^{+}$the trajectory $X_{0}(\cdot)$ admits an extension on the interval $\left[t_{0}, t_{0}+j\right]$ to a $\tilde{G}$-trajectory $X_{j}(\cdot)$ satisfying $(26)$ on $\left[t_{0}, t_{0}+j\right]$. We proceed by the induction argument on $j \in \mathbb{N}^{+}$. Let $j \in \mathbb{N}^{+}$and suppose that $X_{j}(\cdot)=\left(x_{j}(\cdot), v_{j}(\cdot)\right)$ satisfies the claim. Then, using (23) and applying again [12, Corollary 3.2] on the time interval $\left[t_{0}+j, t_{0}+j+1\right]$, we can find a $\tilde{G}$-trajectory $X(\cdot)=(x(\cdot), v(\cdot))$ satisfying

$$
\left\{\begin{array}{l}
X^{\prime}(t) \in \tilde{G}(t, X(t)) \quad \text { for a.e. } t \in\left[t_{0}+j, t_{0}+j+1\right] \\
x\left(\left[t_{0}+j, t_{0}+j+1\right]\right) \subset A \\
x\left(t_{0}+j\right)=x_{j}\left(t_{0}+j\right), v\left(t_{0}+j\right)=v_{j}\left(t_{0}+j\right) \\
v(t) \geqslant W(t, x(t)) \quad \forall t \in\left[t_{0}+j, t_{0}+j+1\right] .
\end{array}\right.
$$

Putting $X_{j+1}(t)=\left(x_{j}(t), v_{j}(t)\right)$ if $t \in\left[t_{0}, t_{0}+j\right]$ and $X_{j+1}(t)=(x(t), v(t))$ if $t \in\left(t_{0}+\right.$ $\left.j, t_{0}+j+1\right]$, we deduce that $X_{j+1}(\cdot)$ satisfies our claim. Now, consider the $\tilde{G}$-trajectory $X(t)=(x(t), v(t))$ given by

$$
X(t)=X_{j}(t) \quad \text { if } t \in\left[t_{0}+j, t_{0}+j+1\right] .
$$

By the measurable selection theorem, there exist two measurable functions $u(\cdot)$ and $r(\cdot)$, with $u(t) \in U(t)$ and $r(t) \in[0, c(t)(1+|x(t)|)-L(t, x(t), u(t))]$ for a.e. $t \geqslant t_{0}$, such that $v(t)=W\left(t_{0}, x_{0}\right)-\int_{t_{0}}^{t} L(s, x(s), u(s)) d s-\int_{t_{0}}^{t} r(s) d s$ for all $t \geqslant t_{0}$. Then

$$
W\left(t_{0}, x_{0}\right) \geqslant W(t, x(t))+\int_{t_{0}}^{t} L(s, x(s), u(s)) d s \quad \forall t \geqslant t_{0}
$$

Thus $(t, x(t)) \in \operatorname{dom} W$ for all $t \geqslant t_{0}$. Since $L(t, \cdot, \cdot) \geqslant \phi(t)$ for a.e. $t \geqslant 0$, where $\phi \in$ $L^{1}([0, \infty) ; \mathbb{R})$, it follows that the $\operatorname{limit} \lim _{t \rightarrow \infty} \int_{t_{0}}^{t} L(s, x(s), u(s)) d s$ exists. So, using $(8)$ and passing to the limit in (27) as $t \rightarrow \infty$ yields $W\left(t_{0}, x_{0}\right) \geqslant \int_{t_{0}}^{\infty} L(s, x(s), u(s)) d s$. Therefore $W\left(t_{0}, x_{0}\right) \geqslant V\left(t_{0}, x_{0}\right)$. Consequently $W \geq V$. 
We show next that $W\left(t_{0}, x_{0}\right) \leqslant V\left(t_{0}, x_{0}\right)$ for all $\left(t_{0}, x_{0}\right) \in[0, \infty) \times A$. If $V\left(t_{0}, x_{0}\right)=+\infty$, then $V\left(t_{0}, x_{0}\right) \geqslant W\left(t_{0}, x_{0}\right)$. So, let us assume that $\left(t_{0}, x_{0}\right) \in \operatorname{dom} V$. Fix $\varepsilon>0$. By our assumptions, there exists $T^{\prime}>t_{0} \operatorname{such}$ that $\operatorname{dom} V(t, \cdot) \subset \operatorname{dom} W(t, \cdot)$ for all $t \geqslant T^{\prime}$ and

$$
\sup _{y \in \operatorname{dom} W(t, \cdot)}|W(t, y)| \leqslant \varepsilon \quad \forall t \geqslant T^{\prime} .
$$

Let $(\bar{x}(\cdot), \bar{u}(\cdot))$ be an optimal trajectory-control pair at $\left(t_{0}, x_{0}\right)$ and consider $s_{i} \uparrow+\infty$ with $\left\{s_{i}\right\}_{i} \subset\left(T^{\prime}, \infty\right)$. Put $\bar{X}(\cdot)=(\bar{x}(\cdot), \bar{z}(\cdot))$ where $\bar{z}(t)=-\int_{t_{0}}^{t} L(s, \bar{x}(s), \bar{u}(s)) d s$. For all $(t, x, w) \in[0, \infty) \times \mathbb{R}^{n} \times \mathbb{R}$ define

$$
Q(t, x, w):=\{(f(t, x, u), L(t, x, u)): u \in U(t)\} .
$$

Applying [6, Theorem 2] we deduce that for any $i$ there exists a $Q$-trajectory $X_{i}(\cdot)=$ $\left(x_{i}(\cdot), z_{i}(\cdot)\right)$ solving

$$
\begin{cases}X_{i}^{\prime}(t) \in Q\left(t, X_{i}(t)\right) & \text { for a.e. } t \in\left[t_{0}, s_{i}\right] \\ X_{i}\left(s_{i}\right)=\left(\bar{x}\left(s_{i}\right), \bar{z}\left(s_{i}\right)\right) & \\ x_{i}(t) \in \operatorname{int} A & \forall t \in\left[t_{0}, s_{i}\right)\end{cases}
$$

and

$$
\lim _{i \rightarrow \infty}\left\|X_{i}-\bar{X}\right\|_{\infty,\left[t_{0}, s_{i}\right]}=0 .
$$

Hence, by the measurable selection theorem, for any $i$ there exists a measurable selection $u_{i}(t) \in U(t)$ such that $\left(x_{i}(\cdot), u_{i}(\cdot)\right)$ satisfies

$$
\begin{aligned}
& \begin{cases}x_{i}^{\prime}(t)=f\left(t, x_{i}(t), u_{i}(t)\right) & \text { for a.e. } t \in\left[t_{0}, s_{i}\right] \\
x_{i}\left(s_{i}\right)=\bar{x}\left(s_{i}\right) & \forall t \in\left[t_{0}, s_{i}\right),\end{cases} \\
& \lim _{i \rightarrow \infty} x_{i}\left(t_{0}\right)=\bar{x}\left(t_{0}\right),
\end{aligned}
$$

and

$$
\lim _{i \rightarrow \infty} \int_{t_{0}}^{s_{i}} L\left(s, x_{i}(s), u_{i}(s)\right) d s=\int_{t_{0}}^{\infty} L(s, \bar{x}(s), \bar{u}(s)) d s .
$$

Now, fix $i \in \mathbb{N}^{+}$and consider $\left\{\tau_{j}\right\}_{j} \subset\left(T^{\prime}, s_{i}\right)$ with $\tau_{j} \rightarrow s_{i}$. Note that, by the dynamic programming principle, $x_{i}\left(\tau_{j}\right) \in \operatorname{dom} V\left(\tau_{j}, \cdot\right)$ for all $j$. Consider the solution $w_{j}(\cdot)$ of the Cauchy problem

$$
\left\{\begin{array}{l}
w^{\prime}(t)=-L\left(t, x_{i}(t), u_{i}(t)\right) \quad \text { for a.e. } t \in\left[t_{0}, \tau_{j}\right] \\
w\left(\tau_{j}\right)=W\left(\tau_{j}, x_{i}\left(\tau_{j}\right)\right) .
\end{array}\right.
$$

From Lemma 4.6, we conclude that

$$
\int_{t_{0}}^{\tau_{j}} L\left(s, x_{i}(s), u_{i}(s)\right) d s+W\left(\tau_{j}, x_{i}\left(\tau_{j}\right)\right) \geqslant W\left(t_{0}, x_{i}\left(t_{0}\right)\right) \quad \forall j .
$$

Hence, by (28),

$$
\int_{t_{0}}^{\tau_{j}} L\left(s, x_{i}(s), u_{i}(s)\right) d s+\varepsilon \geqslant W\left(t_{0}, x_{i}\left(t_{0}\right)\right) \quad \forall j
$$


and taking the limit as $j \rightarrow \infty$ we get $\int_{t_{0}}^{s_{i}} L\left(s, x_{i}(s), u_{i}(s)\right) d s+\varepsilon \geqslant W\left(t_{0}, x_{i}\left(t_{0}\right)\right)$. Passing now to the lower limit as $i \rightarrow \infty$, using (29), (30), and the lower semicontinuity of $W$, we have $\int_{t_{0}}^{\infty} L(s, \bar{x}(s), \bar{u}(s)) d s+\varepsilon \geqslant W\left(t_{0}, x_{0}\right)$, i.e., $V\left(t_{0}, x_{0}\right)+\varepsilon \geqslant W\left(t_{0}, x_{0}\right)$. Since $\varepsilon$ is arbitrary, we conclude that $V\left(t_{0}, x_{0}\right) \geqslant W\left(t_{0}, x_{0}\right)$. Hence $V=W$ on $(0, \infty) \times A$.

Since $t \rightsquigarrow$ epi $W(t, \cdot)$ is locally absolutely continuous and $W$ is lower semicontinuous, $\liminf _{s \rightarrow 0+, y \rightarrow{ }_{A} x} W(s, y)=W(0, x)$ for all $x \in A$. So, fix $x_{0} \in A$. From (12) and what precede, we have

$$
W\left(0, x_{0}\right)=\liminf _{s \rightarrow 0+, y \rightarrow{ }_{A} x_{0}} W(s, y)=\liminf _{s \rightarrow 0+, y \rightarrow{ }_{A} x_{0}} V(s, y)=V\left(0, x_{0}\right) .
$$

Now, assume in addition (B). Let $\bar{t} \in[0, \infty)$ be such that $\operatorname{dom} V(\bar{t}, \cdot) \neq \emptyset$. By (OPC) this implies that $\operatorname{dom} V(t, \cdot) \neq \emptyset$ for all $t \in[0, \bar{t}]$. Moreover, by the dynamic programming principle, it follows that $\operatorname{dom} V(s, \cdot) \neq \emptyset$ for all $s \geqslant \bar{t}$. Hence,

$$
|V(s, y)| \leqslant \int_{s}^{\infty} \psi(\xi) d \xi \quad \forall y \in \operatorname{dom} V(s, \cdot), \forall s \geqslant T
$$

So, we deduce that $V$ satisfies (8).

\section{Lipschitz Continuous Solutions}

In [6] we provided sufficient conditions for the local Lipschitz continuity of the value function under state constraints. Before stating an existence and uniqueness result for Lipschitz continuous solutions (in the Crandall-Lions sense) of H-J-B equation, we show a geometric result (in the spirit of Section 3) involving the hypographs of functions.

Proposition 5.1. Under all the assumptions of Theorem 3.3 suppose that the set-valued map

$$
t \rightsquigarrow\{(x, v) \in A \times \mathbb{R}: v \leqslant W(t, x) \neq+\infty\}
$$

is locally absolutely continuous.

Then the following statements are equivalent:

(i) $W=V$;

(ii) there exists a set $C^{\prime} \subset(0, \infty)$, with $\mu\left(C^{\prime}\right)=0$, such that for all $(t, x) \in \operatorname{dom} W \cap$ $\left(\left((0, \infty) \backslash C^{\prime}\right) \times A\right)$

$$
-p_{t}+H\left(t, x,-p_{x},-q\right) \geqslant 0 \quad \forall\left(p_{t}, p_{x}, q\right) \in T_{\text {epi } W}(t, x, W(t, x))^{-},
$$

for all $(t, x) \in \operatorname{dom} W \cap\left(\left((0, \infty) \backslash C^{\prime}\right) \times \operatorname{int} A\right)$

$$
-p_{t}+H\left(t, x,-p_{x},-q\right) \leqslant 0 \quad \forall\left(p_{t}, p_{x}, q\right) \in T_{\text {hypo } W}(t, x, W(t, x))^{+},
$$

and $t \rightsquigarrow$ epi $W(t, \cdot)$ is locally absolutely continuous. 
Proof. Notice first of all that by the definition of locally absolutely continuous set-valued map, the hypograph of $W(t, \cdot)$ restricted to dom $W(t, \cdot)$ is closed. Assume $(i)$. From Proposition 4.4-(iv), we can find a subset $C \subset(0, \infty)$, with $\mu(C)=0$, such that for any $\left(t_{0}, x_{0}\right) \in((0, \infty) \backslash C) \times \operatorname{int} A$ we have $-L\left(t_{0}, x_{0}, u_{0}\right) \leqslant D_{\downarrow} V\left(t_{0}, x_{0}\right)\left(1, f\left(t_{0}, x_{0}, u_{0}\right)\right)$ for all $u_{0} \in U\left(t_{0}\right)$, i.e., recalling $(1)$,

$$
\left(1, f\left(t_{0}, x_{0}, u_{0}\right),-L\left(t_{0}, x_{0}, u_{0}\right)\right) \in T_{\text {hypo } V}\left(t_{0}, x_{0}, V\left(t_{0}, x_{0}\right)\right) \quad \forall u_{0} \in U\left(t_{0}\right) .
$$

So,

$$
-p_{t}+H\left(t, x,-p_{x},-q\right) \leqslant 0 \quad \forall\left(p_{t}, p_{x}, q\right) \in T_{\text {hypo } V}(t, x, V(t, x))^{+} .
$$

The first inequality in (ii) follows from Theorem 3.3.

Now assume $(i i)$. By Theorem 3.3 and the proof of $(i i) \Longrightarrow(i)$ of Theorem 3.3, it is just sufficient to show (25). Arguing as in the proof of Proposition 4.4-(iii), there exists $C^{\prime} \subset(0, \infty)$, with $\mu\left(C^{\prime}\right)=0$, such that for any $\left(t_{0}, x_{0}\right) \in\left((0, \infty) \backslash C^{\prime}\right) \times \operatorname{int} A$ and $u_{0} \in U\left(t_{0}\right)$, we can find $t_{1} \in\left(0, t_{0}\right)$ and a trajectory-control pair $((x, v),(u, r))(\cdot)$ satisfying $(17)$ and $x\left(\left[t_{1}, t_{0}\right]\right) \subset \operatorname{int} A$. By Lemma 4.8, taking $\left\{s_{i}\right\}_{i} \subset\left(t_{1}, t_{0}\right)$ with $s_{i} \rightarrow t_{0}-$, we get that for all $i$ the solution $w_{i}(\cdot)$ of

$$
\left\{\begin{array}{l}
w^{\prime}(t)=-L(t, x(t), u(t)) \text { for a.e. } t \in\left[s_{i}, t_{0}\right] \\
w\left(s_{i}\right)=W\left(s_{i}, x\left(s_{i}\right)\right)
\end{array}\right.
$$

satisfies $w_{i}\left(t_{0}\right)=W\left(s_{i}, x\left(s_{i}\right)\right)-\int_{s_{i}}^{t_{0}} L(s, x(s), u(s)) d s \leqslant W\left(t_{0}, x\left(t_{0}\right)\right)$. Hence $W\left(s_{i}, x\left(s_{i}\right)\right)-$ $W\left(t_{0}, x_{0}\right) \leqslant \int_{s_{i}}^{t_{0}} L(s, x(s), u(s)) d s \leqslant v\left(s_{i}\right)$ for all $i$. Dividing by $t_{0}-s_{i}$ and passing to the lower limit as $i \rightarrow \infty$, we have the conclusion.

Remark 5.2. Assuming further that $f, L$, and $W:[0, \infty) \times A \rightarrow \mathbb{R}$ are continuous functions, then, using the same arguments as in the proofs of [10, Theorem 4.3 and Lemma 4.3], the assumption (31) in Proposition 5.1 can be skipped and $(i)$ is equivalent to the following:

$$
\begin{cases}-p_{t}+\mathscr{H}\left(t, x,-p_{x}\right) \geqslant 0 & \forall(t, x) \in(0, \infty) \times A, \forall\left(p_{t}, p_{x}\right) \in \partial_{-} W(t, x) \\ -p_{t}+\mathscr{H}\left(t, x,-p_{x}\right) \leqslant 0 & \forall(t, x) \in(0, \infty) \times \operatorname{int} A, \forall\left(p_{t}, p_{x}\right) \in \partial_{+} W(t, x) .\end{cases}
$$

From Theorem 3.3 and Proposition 5.1 we get immediately the following three corollaries.

Corollary 5.3. Assume (h) $)^{\prime \prime}$ and (OPC). Let $W:[0, \infty) \times A \rightarrow \mathbb{R} \cup\{+\infty\}$ be a lower semicontinuous function such that $\operatorname{dom} V(t, \cdot) \subset \operatorname{dom} W(t, \cdot) \neq \emptyset$ for all large $t>0$ and (8) holds true. Suppose that

$\mu\left\{t \in[0, \infty): \exists x \in A,(t, x) \in \operatorname{dom} W,\{0\} \neq T_{\text {epi } W}(t, x, W(t, x))^{-} \subset \mathbb{R} \times \mathbb{R}^{n} \times\{0\}\right\}=0$.

Then the following statements are equivalent:

(i) $W=V$; 
(ii) there exists a set $C^{\prime} \subset(0, \infty)$, with $\mu\left(C^{\prime}\right)=0$, satisfying for all $(t, x) \in \operatorname{dom} W \cap$ $\left(\left((0, \infty) \backslash C^{\prime}\right) \times \partial A\right)$

$$
-p_{t}+\mathscr{H}\left(t, x,-p_{x}\right) \geqslant 0 \quad \forall\left(p_{t}, p_{x}\right) \in \partial_{-} W(t, x),
$$

for all $(t, x) \in \operatorname{dom} W \cap\left(\left((0, \infty) \backslash C^{\prime}\right) \times \operatorname{int} A\right)$

$$
-p_{t}+\mathscr{H}\left(t, x,-p_{x}\right)=0 \quad \forall\left(p_{t}, p_{x}\right) \in \partial_{-} W(t, x),
$$

and $t \rightsquigarrow$ epi $W(t, \cdot)$ is locally absolutely continuous.

Corollary 5.4. Under all the assumptions of Corollary 5.3 suppose that the set-valued map

$$
t \rightsquigarrow\{(x, v) \in A \times \mathbb{R}: v \leqslant W(t, x) \neq+\infty\},
$$

is locally absolutely continuous and

$\mu\left\{t \in[0, \infty): \exists x \in A,(t, x) \in \operatorname{dom} W,\{0\} \neq T_{\text {hypo } W}(t, x, W(t, x))^{+} \subset \mathbb{R} \times \mathbb{R}^{n} \times\{0\}\right\}=0$.

Then the following statements are equivalent:

(i) $W=V$;

(ii) there exists a set $C^{\prime} \subset(0, \infty)$, with $\mu\left(C^{\prime}\right)=0$, satisfying for all $(t, x) \in \operatorname{dom} W \cap$ $\left(\left((0, \infty) \backslash C^{\prime}\right) \times A\right)$

$$
-p_{t}+\mathscr{H}\left(t, x,-p_{x}\right) \geqslant 0 \quad \forall\left(p_{t}, p_{x}\right) \in \partial_{-} W(t, x),
$$

for all $(t, x) \in \operatorname{dom} W \cap\left(\left((0, \infty) \backslash C^{\prime}\right) \times \operatorname{int} A\right)$

$$
-p_{t}+\mathscr{H}\left(t, x,-p_{x}\right) \leqslant 0 \quad \forall\left(p_{t}, p_{x}\right) \in \partial_{+} W(t, x),
$$

and $t \rightsquigarrow$ epi $W(t, \cdot)$ is locally absolutely continuous.

Remark 5.5. Let $W:[0, \infty) \times A \rightarrow \mathbb{R}$ be a locally Lipschitz continuous function. Then it is well known that

$$
\left\{\begin{array}{l}
0 \neq\left(p_{t}, p_{x}, q\right) \in T_{\text {epi } W}(t, x, W(t, x))^{-} \Longrightarrow q \neq 0 \\
0 \neq\left(p_{t}, p_{x}, q\right) \in T_{\text {hypo } W}(t, x, W(t, x))^{+} \Longrightarrow q \neq 0 .
\end{array}\right.
$$

and if $\partial_{-} W(t, x) \neq \emptyset$, then $T_{\text {epi } W}(t, x, W(t, x))^{-}=\cup_{\lambda \geqslant 0} \lambda\left(\partial_{-} W(t, x),-1\right)$. Similarly, if $\partial_{+} W(t, x) \neq \emptyset$, then $T_{\text {hypo } W}(t, x, W(t, x))^{+}=\cup_{\lambda \geqslant 0} \lambda\left(\partial_{+} W(t, x),-1\right)$.

From Corollary 5.4 and Remark 5.5, we deduce the following:

Corollary 5.6. Assume (h)" and (OPC). Let $W:[0, \infty) \times A \rightarrow \mathbb{R}$ be a locally Lipschitz continuous function satisfying (8). Then the following statements are equivalent:

(i) $W=V$; 
(ii) there exists a set $C^{\prime} \subset(0, \infty)$, with $\mu\left(C^{\prime}\right)=0$, satisfying for all $(t, x) \in \operatorname{dom} W \cap$ $\left(\left((0, \infty) \backslash C^{\prime}\right) \times A\right)$

$$
-p_{t}+\mathscr{H}\left(t, x,-p_{x}\right) \geqslant 0 \quad \forall\left(p_{t}, p_{x}\right) \in \partial_{-} W(t, x),
$$

for all $(t, x) \in \operatorname{dom} W \cap\left(\left((0, \infty) \backslash C^{\prime}\right) \times \operatorname{int} A\right)$

$$
-p_{t}+\mathscr{H}\left(t, x,-p_{x}\right) \leqslant 0 \quad \forall\left(p_{t}, p_{x}\right) \in \partial_{+} W(t, x) .
$$

Now, let $l:[0, \infty) \times \mathbb{R}^{n} \times \mathbb{R}^{m} \rightarrow[0, \infty)$ be a bounded measurable function, $\lambda>0$, and

$$
L(t, x, u)=e^{-\lambda t} l(t, x, u)
$$

Proposition 5.7. Assume (32), (h)", and (IPC).

Then, there exists $\bar{\lambda}>0$ such that for all $\lambda \geqslant \bar{\lambda}$ the value function $V$ is the unique locally Lipschitz continuous function on $[0, \infty) \times A$ satisfying

$$
\left\{\begin{array}{l}
-p_{t}+\mathscr{H}\left(t, x,-p_{x}\right) \geqslant 0 \forall\left(p_{t}, p_{x}\right) \in \partial_{-} V(t, x), \text { for a.e. } t>0, \forall x \in A \\
-p_{t}+\mathscr{H}\left(t, x,-p_{x}\right) \leqslant 0 \forall\left(p_{t}, p_{x}\right) \in \partial_{+} V(t, x), \text { for a.e. } t>0, \forall x \in \operatorname{int} A, \\
\lim _{t \rightarrow \infty} \sup _{y \in A}|V(t, y)|=0 .
\end{array}\right.
$$

Proof. From [6, Theorem 4] and the proof of [6, Corollary 1] it follows that there exists $\bar{\lambda}>0$ such that for all $\lambda \geqslant \bar{\lambda}$ the value function $V$ is locally Lipschitz continuous on $[0, \infty) \times A$. Moreover, arguing as in the proofs $(i) \Longrightarrow(i i)$ of Theorem 3.3 and Proposition 5.1, and from Remarks 3.1-(i) and 5.5, we deduce that $V$ satisfies (33).

Now, let $W:[0, \infty) \times A \rightarrow \mathbb{R}$ be a locally Lipschitz continuous function satisfying (33). From the proof $(i i) \Longrightarrow(i)$ of Theorem 3.3 it follows that $W \geqslant V$ on $(0, \infty) \times A$. Let $\left(t_{0}, x_{0}\right) \in(0, \infty) \times A,(\bar{x}(\cdot), \bar{u}(\cdot))$ be optimal at $\left(t_{0}, x_{0}\right)$, and $\varepsilon>0, T^{\prime}>t_{0}$ such that $(28)$ holds true. Consider $s_{i} \uparrow+\infty$ with $\left\{s_{i}\right\}_{i} \subset\left(T^{\prime}, \infty\right)$. Fix $i \in \mathbb{N}^{+}$and let $\left\{\tau_{j}\right\}_{j} \subset\left(t_{0}, s_{0}\right)$ and $\left\{y_{j}\right\}_{j} \subset \operatorname{int} A$ be such that $\tau_{j} \rightarrow t_{0}$ and $y_{j} \rightarrow x_{0}$. Repeating the same arguments as in the proof of the implication $(i i) \Longrightarrow(i)$ of Theorem 3.3 and using [6, Theorem 2], we show that for all $j$ there exists a measurable selection $u_{j}(\cdot) \in U(\cdot)$ on $\left[\tau_{j}, s_{i}\right]$ such that $\left(x_{j}(\cdot), u_{j}(\cdot)\right)$ satisfies

$$
\begin{array}{cl}
\left\{\begin{array}{ll}
x_{j}^{\prime}(t)=f\left(t, x_{j}(t), u_{j}(t)\right) & \text { for a.e. } t \in\left[\tau_{j}, s_{i}\right] \\
x_{j}\left(\tau_{j}\right)=y_{j} & \forall t \in\left[\tau_{j}, s_{i}\right] \\
x_{j}(t) \in \operatorname{int} A & \lim _{j \rightarrow \infty}\left\|x_{j}-\bar{x}\right\|_{\infty,\left[\tau_{j}, s_{i}\right]}
\end{array}=0\right.
\end{array}
$$

and

$$
\lim _{j \rightarrow \infty} \int_{\tau_{j}}^{s_{i}} L\left(s, x_{j}(s), u_{j}(s)\right) d s=\int_{t_{0}}^{s_{i}} L(s, \bar{x}(s), \bar{u}(s)) d s .
$$

Consider the solution $w_{j}(\cdot)$ of the Cauchy problem

$$
\left\{\begin{array}{l}
w^{\prime}(t)=-L\left(t, x_{j}(t), u_{j}(t)\right) \quad \text { for a.e. } t \in\left[\tau_{j}, s_{i}\right] \\
w\left(\tau_{j}\right)=W\left(\tau_{j}, y_{j}\right)
\end{array}\right.
$$


From Lemma 4.8 we get

$$
W\left(\tau_{j}, y_{j}\right)-\int_{\tau_{j}}^{s_{i}} L\left(s, x_{j}(s), u_{j}(s)\right) d s \leqslant W\left(s_{i}, x_{j}\left(s_{i}\right)\right) \quad \forall j .
$$

So, by (28), passing to the limit as $j \rightarrow \infty$, using (34), (35), and the continuity of $W$, we have $W\left(t_{0}, x_{0}\right) \leqslant \int_{t_{0}}^{s_{i}} L(s, \bar{x}(s), \bar{u}(s)) d s+\varepsilon$. Then, passing to the limit as $i \rightarrow \infty$ and since $\varepsilon$ is arbitrarily small, we get $W\left(t_{0}, x_{0}\right) \leqslant V\left(t_{0}, x_{0}\right)$.

Finally, since $V=W$ on $(0, \infty) \times A$, from the continuity of $V$ and $W$, the conclusion follows.

\section{Appendix}

Proof of Lemma 4.6. Notice that, by the separation theorem, (18) is equivalent to $\{-1\} \times$ $-G(t, x) \subset \overline{\operatorname{co}} T_{\text {epi } W}(t, x, v)$ for all $v \geqslant W(t, x)$ and all $(t, x) \in\left(\left((0, \infty) \backslash C^{\prime}\right) \times \operatorname{int} A\right) \cap \operatorname{dom} W$. Let $0<\tau_{0}<\tau_{1}$. Thus

$$
(1, \tilde{f}(s, x, u), \tilde{L}(s, x, u)) \in \overline{\operatorname{co}} T_{\operatorname{graph} Q}(s, x, v)
$$

for a.e. $s \in\left[0, \tau_{1}-\tau_{0}\right]$, any $(x, v) \in Q(s) \cap(\operatorname{int} A \times \mathbb{R})$, and any $u \in U(s)$, where $\tilde{f}(s, x, u):=$ $-f\left(\tau_{1}-s, x, u\right), \tilde{L}(s, x, u):=L\left(\tau_{1}-s, x, u\right)$, and $Q(s):=$ epi $W\left(\tau_{1}-s, \cdot\right)$. Consider a trajectory-control pair $(x(\cdot), u(\cdot))$ solving (3) on $I=\left[\tau_{0}, \tau_{1}\right]$, with $x\left(\left[\tau_{0}, \tau_{1}\right]\right) \subset \operatorname{int} A$ and $\left(\tau_{1}, x\left(\tau_{1}\right)\right) \in \operatorname{dom} W$. Putting $\tilde{u}(\cdot)=u\left(\tau_{1}-\cdot\right)$, we claim that $d_{Q(s)}((y(s), \tilde{w}(s)))=0$ for all $s \in\left[0, \tau_{1}-\tau_{0}\right]$, where $y(\cdot)=x\left(\tau_{1}-\cdot\right)$ and $\tilde{w}(\cdot)=w\left(\tau_{1}-\cdot\right)$ are the unique solutions of $y^{\prime}(s)=$ $\tilde{f}(s, y(s), \tilde{u}(s))$ and $\tilde{w}^{\prime}(s)=\tilde{L}(s, y(s), \tilde{u}(s))$ a.e. $s \in\left[0, \tau_{1}-\tau_{0}\right]$, respectively, with $y(0)=$ $x\left(\tau_{1}\right)$ and $\tilde{w}(0)=W\left(\tau_{1}, x\left(\tau_{1}\right)\right)$. Putting $g(s)=d_{Q(s)}((y(s), \tilde{w}(s)))$, from [15, Lemma 4.8], applied to the single-valued map $s \rightsquigarrow\{(\tilde{f}(s, y(s), \tilde{u}(s)), \tilde{L}(s, y(s), \tilde{u}(s)))\}$, it follows that $g(\cdot)$ is absolutely continuous. Pick $(\xi(s), r(s)) \in Q(s)$ with $g(s)=|(y(s), \tilde{w}(s))-(\xi(s), r(s))|$ for all $s \in\left[0, \tau_{1}-\tau_{0}\right]$. We claim that $g(\cdot) \equiv 0$ on $\left(0, \tau_{1}-\tau_{0}\right]$. Indeed, otherwise, we can find $T \in\left(0, \tau_{1}-\tau_{0}\right]$ with $g(T)>0$. Denoting $t^{*}=\sup \{t \in[0, T]: g(t)=0\}$, let $\varepsilon>0$ be such that $\xi(s) \in \operatorname{int} A$ and $g(s)>0$ for any $s \in\left(t^{*}, t^{*}+\varepsilon\right]$. Consider $s \in\left(t^{*}, t^{*}+\varepsilon\right)$ where $g(\cdot)$, $y(\cdot)$, and $\tilde{w}(\cdot)$ are differentiable, with $y^{\prime}(s)=\tilde{f}(s, y(s), \tilde{u}(s))$ and $\tilde{w}^{\prime}(s)=\tilde{L}(s, y(s), \tilde{u}(s))$. Let $(\theta, v) \in T_{\operatorname{graph} Q}(s, \xi(s), r(s))$ and $\theta_{i} \rightarrow \theta, v_{i} \rightarrow v, h_{i} \rightarrow 0+$ satisfy

$$
(\xi(s), r(s))+h_{i} v_{i} \in Q\left(s+h_{i} \theta_{i}\right) \quad \forall i .
$$

Then, setting $Z=(y(s), \tilde{w}(s))$ and $Y=(\xi(s), r(s))$, we get

$$
g\left(s+h_{i} \theta_{i}\right)-g(s) \leqslant\left|\left(y\left(s+h_{i} \theta_{i}\right), \tilde{w}\left(s+h_{i} \theta_{i}\right)\right)-Y-h_{i} v_{i}\right|-|Z-Y| .
$$

Dividing this inequality by $h_{i}$ and passing to the limit as $i \rightarrow \infty$ we have

$$
g^{\prime}(s) \theta \leqslant\langle p,(\tilde{f}(s, y(s), \tilde{u}(s)), \tilde{L}(s, y(s), \tilde{u}(s))) \theta-v\rangle,
$$

where $p=\frac{Z-Y}{|Z-Y|}$. Since (37) holds for any $(\theta, v) \in T_{\operatorname{graph} Q}(s, \xi(s), r(s))$. Taking convex combinations of elements in $T_{\operatorname{graph} Q}(s, \xi(s), r(s))$ we conclude that (37) holds for all $(\theta, v) \in$ $\overline{\mathrm{co}} T_{\text {graph } Q}(s, \xi(s), r(s))$. By (36) the inequality (37) holds true for

$$
\theta=1 \quad \& \quad v=(\tilde{f}(s, \xi(s), \tilde{u}(s)), \tilde{L}(s, \xi(s), \tilde{u}(s))) .
$$


Therefore $g^{\prime}(s) \leqslant k(s)|y(s)-\xi(s)| \leqslant k(s) g(s)$. From the Gronwall lemma we conclude that $g(\cdot) \equiv 0$ on $\left[t^{*}, t^{*}+\varepsilon\right]$, leading to a contradiction. Thus $g=0$ and the proof is complete.

\section{References}

[1] J.-P. Aubin and H. Frankowska. Set-valued analysis. Modern Birkhäuser Classics. Birkhäuser Boston, Inc., Boston, MA, 2009.

[2] M. Bardi and I. Capuzzo-Dolcetta. Optimal control and viscosity solutions of HamiltonJacobi-Bellman equations. Springer Science \& Business Media, 2008.

[3] G. Barles. Existence results for first order Hamilton Jacobi equations. Ann. Inst. H. Poincaré Anal. Non Linéaire, 1(5):325-340, 1984.

[4] E. N. Barron and R. Jensen. Semicontinuous viscosity solutions for HamiltonJacobi equations with convex Hamiltonians. Comm. Partial Differential Equations, 15(12):1713-1742, 1990.

[5] E. N. Barron and R. Jensen. Optimal control and semicontinuous viscosity solutions. Proc. Amer. Math. Soc., 113(2):397-402, 1991.

[6] V. Basco and H. Frankowska. Lipschitz continuity of the value function for the infinite horizon optimal control problem under state constraints (submitted).

[7] P. Cannarsa and H. Frankowska. Value function, relaxation, and transversality conditions in infinite horizon optimal control. J. Math. Anal. Appl., 457(2):1188-1217, 2018 .

[8] M. G. Crandall, L. C. Evans, and P.-L. Lions. Some properties of viscosity solutions of Hamilton-Jacobi equations. Trans. Amer. Math. Soc., 282(2):487-502, 1984.

[9] M. G. Crandall and P.-L. Lions. Viscosity solutions of Hamilton-Jacobi equations. Trans. Amer. Math. Soc., 277(1):1-42, 1983.

[10] H. Frankowska. Lower semicontinuous solutions of Hamilton-Jacobi-Bellman equations. SIAM J. Control Optim., 31(1):257-272, 1993.

[11] H. Frankowska and M. Mazzola. Discontinuous solutions of Hamilton-Jacobi-Bellman equation under state constraints. Calculus of Variations and Partial Differential Equations, 46(3-4):725-747, 2013.

[12] H. Frankowska and S. Plaskacz. A measurable upper semicontinuous viability theorem for tubes. Nonlinear Analysis: Theory, Methods \& Applications, 26(3):565-582, 1996.

[13] H. Frankowska and S. Plaskacz. Hamilton-Jacobi equations for infinite horizon control problems with state constraints. Procedings of International Conference: Calculus of Variations and Related Topics (Haifa, March 25-April 1, 1998), pages 97-116, 1999.

[14] H. Frankowska and S. Plaskacz. Semicontinuous solutions of Hamilton-Jacobi-Bellman equations with degenerate state constraints. J. Math. Anal. Appl., 251(2):818-838, 2000 . 
[15] H. Frankowska, S. Plaskacz, and T. Rzeżuchowski. Measurable viability theorems and the Hamilton-Jacobi-Bellman equation. J. Differential Equations, 116(2):265-305, 1995 .

[16] H. Frankowska and H. Sedrakyan. Stable representation of convex Hamiltonians. Nonlinear Analysis: Theory, Methods \& Applications, 100:30-42, 2014.

[17] H. Ishii. Hamilton-Jacobi equations with discontinuous Hamiltonians on arbitrary open sets. Bull. Fac. Sci. Engrg. Chuo Univ., 28:33-77, 1985.

[18] H. Ishii. Perron's method for monotone systems of second-order elliptic partial differential equations. Differential Integral Equations, 5(1):1-24, 1992.

[19] H. Ishii and S. Koike. A new formulation of state constraint problems for first-order PDEs. SIAM J. Control Optim., 34(2):554-571, 1996.

[20] P.-L. Lions. Generalized solutions of Hamilton-Jacobi equations, volume 69 of Research Notes in Mathematics. Pitman, 1982.

[21] P.-L. Lions and B. Perthame. Remarks on Hamilton-Jacobi equations with measurable time-dependent Hamiltonians. Nonlinear Anal., 11(5):613-621, 1987.

[22] F. Rampazzo. Faithful representations for convex Hamilton-Jacobi equations. SIAM J. Control Optim., 44(3):867-884, 2005.

[23] R. T. Rockafellar. Proximal subgradients, marginal values, and augmented Lagrangians in nonconvex optimization. Math. Oper. Res., 6(3):424-436, 1981.

[24] R. T. Rockafellar and R. J. B. Wets. Variational analysis, volume 317 of Grundlehren der Mathematischen Wissenschaften. Springer-Verlag, Berlin, 1998.

[25] H. M. Soner. Optimal control problems with state-space constraints I. SIAM J. Control Optim., 24:552-562, 1986.

[26] P. E. Souganidis. Existence of viscosity solutions of Hamilton-Jacobi equations. $J$. Differential Equations, 56(3):345-390, 1985. 